\title{
Phase space structures in gyrokinetic simulations of fusion plasma turbulence
}

\author{
Philippe Ghendrih $^{1}$, Claudia Norscini ${ }^{1}$, Thomas Cartier-Michaud ${ }^{1}$, Guilhem Dif-Pradalier ${ }^{1}$, Jérémie Abiteboul ${ }^{2}$, \\ Yue Dong ${ }^{3}$, Xavier Garbet ${ }^{1}$, Ozgür Gürcan ${ }^{3}$, Pascale Hennequin ${ }^{3}$, Virginie Grandgirard ${ }^{1}$, Guillaume Latu ${ }^{1}$, \\ Pierre Morel $^{3}$, Yanick Sarazin ${ }^{1}$, Alexandre Storelli ${ }^{3}$, and Laure Vermare ${ }^{3}$ \\ 1 CEA, IRFM, F-13108 Saint-Paul-lez-Durance, France. \\ 2 Max-Planck-Institut fur Plasmaphysik, Boltzmannstr. 2, D-85748 Garching, Germany. \\ 3 LPP, Ecole Polytechnique, CNRS, 91128 Palaiseau, France.
}

Received: date / Revised version: date

\begin{abstract}
Gyrokinetic simulations of fusion plasmas give extensive information in 5D on turbulence and transport. This paper highlights a few of these challenging physics in global, flux driven simulations using experimental inputs from Tore Supra shot TS45511. The electrostatic gyrokinetic code GYSELA is used for these simulations. The 3D structure of avalanches indicate that these structures propagate radially at localised toroidal angles and then expand along the field line at sound speed to form the filaments. Analysing the poloidal mode structure of the potential fluctuations (at a given toroidal location), one finds that the low modes $m=0$ and $m=1$ exhibit a global structure; the magnitude of the $m=0$ mode is much larger than that of the $m=1$ mode. The shear layers of the corrugation structures are thus found to be dominated by the $m=0$ contribution, that are comparable to that of the zonal flows. This global mode seems to localise the $m=2$ mode but has little effect on the localisation of the higher mode numbers. However when analysing the pulsation of the latter modes one finds that all modes exhibit a similar phase velocity, comparable to the local zonal flow velocity. The consequent dispersion like relation between the modes pulsation and the mode numbers provides a means to measure the zonal flow. Temperature fluctuations and the turbulent heat flux are localised between the corrugation structures. Temperature fluctuations are found to exhibit two scales, small fluctuations that are localised by the corrugation shear layers, and appear to bounce back and forth radially, and large fluctuations, also readily observed on the flux, which are associated to the disruption of the corrugations. The radial ballistic velocity of both avalanche events if of the order of $0.5 \rho_{*} c_{0}$ where $\rho_{*}=\rho_{0} / a, a$ being the tokamak minor radius and $\rho_{0}$ being the characteristic Larmor radius, $\rho_{0}=c_{0} / \Omega_{0} . c_{0}$ is the reference ion thermal velocity and $\Omega_{0}=q_{i} B_{0} / m_{i}$ the reference ion Larmor frequency for the characteristic amplitude of the magnetic field $B_{0}, q_{i}$ and $m_{i}$ being respectively the ion charge and mass. The electric drift velocity is also found to exhibit a poloidal pattern, with maximum amplitude of the fluctuations either in the top or in the bottom regions of the machine depending on the sign of the zonal flow shear. This effect is found to be correlated to the stopping capability of the corrugation structures. The neoclassical properties stemming from the trapped particle drifts lead to large distortion of the distribution function. As expected, these prevail at the outer part of the simulation region despite the large collisionality. The distribution function fluctuations appear to be aligned along the $v_{\|}=$constant lines at constant poloidal angle. A specific symmetry is observed regarding the interplay of turbulence with the trapped-passing region.
\end{abstract}

PACS. 05.10.Gg Stochastic analysis methods - 05.65.+b Self-organized systems - 52.65.Tt Gyrofluid and gyrokinetic simulations - 52.25.Fi Transport properties - 52.25.Xz Magnetized plasmas - 52.25.Gj Fluctuation and chaos phenomena - 52.30.-q Plasma dynamics and flow - 52.35.Ra Plasma turbulence

\section{Introduction}

Magnetic fusion devices aim at confining high temperature plasmas,thermal energy in the range of $10 \mathrm{keV}$, and at relatively low density, in the range of $10^{20} \mathrm{~m}^{-3}$ [1]. These plasmas exhibit a low collisionality so that the correct representation of plasma as a continuous medium is the one particle distribution function. The evolution of the latter is then governed by the Vlasov equation. It is found that the limitation in plasma confinement by the large magnetic field is due to turbulence which is observed to be characterised by scales of the order of the ion Larmor radius and frequencies significantly lower than the Larmor gyration frequency [2]. 
In such a framework, one readily finds that the asymptotic limits of quasineutrality and independence of the distribution function on the Larmor gyration angle of the particle motion. The former condition, via the equality of the ion and electron density, yields a Poisson like equation determining the electric potential in the case of electrostatic turbulence. The latter condition allows one to perform gyro-averages, averages on the Larmor gyration motion, leading to the gyrokinetic framework in 5-D [3], since the gyration angle is not relevant, hence 3 -D for the physical space, 1-D for the velocity parallel to the magnetic field and 1-D for the magnetic moment $\mu$, the invariant conjugate to the gyro-angle [3]. Consistently, the Vlasov equation is then modified and the gyrokinetic equation is considered where the transverse motion is governed by drift velocities [3].

We consider here ion heat transport governed by the so-called Ion Temperature Gradient (ITG) turbulence with adiabatic electrons and in the electrostatic limit. The nonlinear gyrokinetic evolution equation is solved with the GYSELA code [4] with no scale separation, hence for the full distribution function and the geometry of a full or a large fraction of the toroidal annulus (in contrast to flux tube geometries). A symmetry condition on the distribution function, hence with zero heat flux, is used at the inner boundary. The outer boundary condition is a thermal bath with a Maxwellian distribution at given temperature. Towards the inner boundary a heat source is implemented. A constant heat flux is thus imposed to the system [5]. In statistical steady state the mean heat outflux at the outer boundary balances the heat source. With these conditions, the plasma temperature profile evolves freely. Conversely, the adiabatic electron response enforces a constant density gradient [2].

A remarkable feature of these flux-driven simulation is the self-organisation of the turbulence that couples all available scales from the size of the device, typically the minor radius of the torus $a$, to the size of the turbulent cells, of the order of the ion Larmor radius $\rho_{i}$. An outstanding issue is that of turbulence self regulation with on the one hand long range radial transport [6-9] and on the other hand zonal flows [10]. These combine in a selfconsistent way to determine the level of turbulent transport. Ion energy confinement, as addressed in this paper, is then characterised by the mean ion temperature gradient that can be achieved for a given mean heat flux.

The large scale transport events exhibit several features of avalanches as addressed in Self-Organised Criticality [11]. They have been reported in fluid turbulence modelling $[6,8,12]$ as well as gyrokinetics modelling $[5,13$ 16]. The amount of data generated by the flux driven, "full-torus", 5-D gyrokinetic codes is so large that only a fraction is actually stored. A more complex analysis of the data, combining various subsets of data, in then required to address the transport properties at microscopic and mesoscopic scales. This is the case when addressing the local features of avalanche transport. Flux surface av- erages have enabled one to identify these large transport events on the basis of their localisation in radius and time. The issue of their localisation on a flux surface, hence poloidally and toroidally is important to resolve the conjunction of ballooned transport and micro-turbulence filaments. Conversely, transport barriers are understood as long lived structures, homogeneous on a magnetic surface and in some cases evolving on macro time scales radially. Their actual properties at micro and meso scales remains to be determined. The present analysis of self-generated micro-barriers is a first step in that direction.

In this paper we address therefore key aspects of the kinetic turbulence self-organisation. In Section 2 we present the equations implemented in the code GYSELA. The simulation conditions are based on the Tore Supra shot TS45511 presented in Section 3. The dynamics of the poloidal modes of the electric potential, computed at given toroidal position, are presented in Section 4. They indicate that the large mode numbers are convected by the flow of the $m=0$ mode akin to the zonal flow. We then analyse the avalanche transport. A first point is the localisation on the magnetic surfaces, Section 5. In Section 6, we investigate the interplay between avalanche transport and micro-barriers, also named corrugations [16]. Finally we analyse the impact of small electrostatic fluctuations and the dynamics governed by trapped particle drifts on the distribution function, Section 7, Discussion and Conclusion, Section 8, close the paper.

\section{Gyrokinetic equations in the GYSELA code}

A large class of micro instabilities in fusion devices can be described in the gyrokinetic formalism, hence assuming that the characteristic frequencies are much smaller than the ion Larmor frequency. The gyro-angle can then be averaged out and its associated action, the magnetic moment $\mu$ is a constant of motion. In this framework, and in the electrostatic limit, the evolution of the distribution function of the guiding centres takes the following form:

$$
\partial_{t} F+\frac{1}{\mathcal{J}} \frac{\partial}{\partial \boldsymbol{X}} \cdot\left(\mathcal{J} \frac{d \boldsymbol{X}}{d t} F\right)=\mathcal{C}+\mathcal{S}+\mathcal{D}
$$

where $\boldsymbol{X}$ is the $4 \mathrm{D}$ phase space position of the guiding centre $\boldsymbol{X}=\left(\boldsymbol{x}, v_{\|}\right), \boldsymbol{x}$ being the position in space and $v_{\|}$ the velocity parallel to the magnetic field $\boldsymbol{B}$. The guiding centre trajectory $d \boldsymbol{X} / d t$ is defined as:

$$
\begin{aligned}
\frac{d x^{i}}{d t} & =\left(v_{\|} \boldsymbol{b}^{*}+\boldsymbol{V}_{E \times B}^{J}+\boldsymbol{V}_{D}\right) \cdot \boldsymbol{\nabla} x^{i} \\
\frac{d v_{\|}}{d t} & =-\frac{\mu \boldsymbol{\nabla} B+q_{i} \boldsymbol{\nabla} \phi^{J}}{m_{i}} \cdot \boldsymbol{b}^{*}+v_{\|} \boldsymbol{V}_{E \times B}^{J} \cdot \frac{\boldsymbol{\nabla} B}{B}
\end{aligned}
$$

the projections of the drift velocities being:

$$
\begin{array}{r}
\boldsymbol{V}_{E \times B}^{J} \cdot \boldsymbol{\nabla} x^{i}=\frac{\boldsymbol{b} \cdot\left(\boldsymbol{\nabla} U^{J} \times \boldsymbol{\nabla} x^{i}\right)}{\mathcal{J}} \\
\boldsymbol{V}_{D} \cdot \boldsymbol{\nabla} x^{i}=\frac{\left(m_{i} v_{\|}^{2}+\mu B\right)}{q_{i} B} \frac{\boldsymbol{b} \cdot\left(\boldsymbol{\nabla} B \times \boldsymbol{\nabla} x^{i}\right)}{\mathcal{J}}
\end{array}
$$


In these expressions the potential $U^{J}$ is the gyro-averaged electrostatic potential, $\phi^{J}=J_{0} U$, where the operator $J_{0}$ is the gyro-angle averaging operator. As a consequence the potential $\phi^{J}$ is $4 \mathrm{D}$ field depending on space as $\phi$ and also on the magnetic moment $\mu$. Finally, one defines the Jacobian $\mathcal{J}=\boldsymbol{B}^{*} \cdot \boldsymbol{b}=B_{\|}^{*}$ where $\boldsymbol{b}=\boldsymbol{B} / B$ and:

$$
\boldsymbol{B}^{*}=B_{\|}^{*} \boldsymbol{b}^{*}=B\left(\boldsymbol{b}+\frac{m_{i} v_{\|}}{q_{i}} \frac{\boldsymbol{J}}{B^{2} / \mu_{0}}\right)
$$

In this equation $\boldsymbol{J}$ is the plasma current. The term depending on $\boldsymbol{J}$, yields in most cases a small correction. All equations and quantities can readily be normalised. The characteristic space scale is chosen to be the reference Larmor radius $\rho_{0}=c_{0} / \Omega_{0}, \Omega_{0}$ being the reference ion cyclotron frequency with characteristic magnitude of the magnetic field $B_{0}, \Omega_{0}=q_{i} B_{0} / m_{i}, q_{i}$ and $m_{i}$ being respectively the ion charge and mass. The time scale is chosen to be $a / c_{0}=1 /\left(\rho_{*} \Omega_{0}\right)$, where $a$ is the plasma minor radius and $c_{0}=\sqrt{T_{0} / m_{i}} . T_{0}$ is the reference ion temperature. The parameter $\rho_{*}$ is defined as the ratio $\rho_{*}=\rho_{0} / a$.

In equation $(1), \mathcal{C}, \mathcal{S}$ and $\mathcal{D}$ are respectively the collision operator $[17,18]$, the source term $[19,20]$ and the diffusion term introduced in the buffer regions at the inner and outer minor radius that define the radial extent of the simulation domain.

Given the scales of interest, the system is closed with the quasineutrality condition. When considering adiabatic electrons, the latter can take the form:

$$
\begin{aligned}
-\frac{1}{n_{0}} \nabla_{\perp} \cdot\left\langle\frac{m_{i} n_{0}}{q_{i} B^{2}} \nabla_{\perp} U\right\rangle_{f s} & =\frac{\langle n\rangle_{f s}}{n_{0}}-1 \\
-\frac{1}{n_{0}} \nabla_{\perp} \cdot\left(\frac{m_{i} n_{0}}{q_{i} B^{2}} \nabla_{\perp} \tilde{U}\right)+\frac{e}{T_{e}} \tilde{U} & =\frac{\tilde{n}}{n_{0}}
\end{aligned}
$$

where $U=\langle U\rangle_{f s}+\tilde{U}$ and $n=\langle n\rangle+\tilde{n}$, the averaged labelled $f s$ is a flux surface average that stems from the electron adiabatic response which is restricted to a field line. The density $n$ is defined in terms of the distribution function $F$ and the gyro-angle averaging operator $J_{0}$ :

$$
n=2 \pi \int d \mu \int d v_{\|} \mathcal{J} J_{0} F
$$

The GYSELA equations must be completed with the magnetic equilibrium. We consider an axisymmetric equilibrium with concentric and circular magnetic surfaces, that are close enough to the actual magnetic equilibrium of the chosen Tore Supra reference shot, hence:

$$
\boldsymbol{B}=B_{0}\left(\frac{R_{0} B_{0}}{B_{0}} \boldsymbol{\nabla} \varphi+\frac{r^{2}}{q R} \nabla \theta\right)
$$

The parameter $q(r)$ is the safety factor that depends on the plasma current profile in the Tokamak and is related to the field line pitch, namely the variation of the angle $\varphi$ with respect to $\theta$ along the field lines:

$$
\left.\frac{d \varphi}{d \theta}\right|_{F . L .}=\frac{\boldsymbol{B} \cdot \nabla \varphi}{\boldsymbol{B} \cdot \nabla \theta}=q \frac{R_{0}}{R}
$$
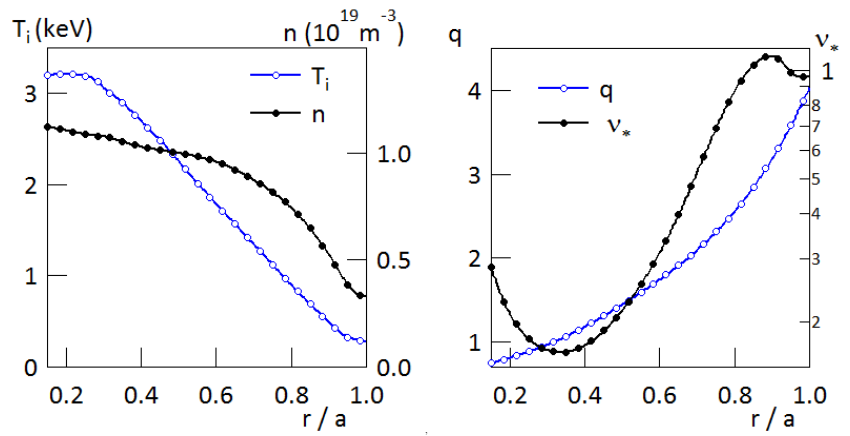

Fig. 1. Profiles used in the simulation, left hand side density $n$ and initial temperature $T_{i}$, right hand side safety factor $\mathrm{q}$ and collisionality $\nu_{*}$.

Since the chosen angles are not magnetic coordinates, one obtains the local field line pitch depending on both $r$ and $\theta$ since $R=R_{0}+r \cos (\theta)$.

\section{Parameters and main features of the simulations}

In this Section we analyse the simulation input for a plasma comparable to Tore Supra shot TS45511, but for devices larger $\rho_{*}$, namely $\rho_{*}=1 / 150$ and $\rho_{*}=1 / 300$ while the Tore Supra shot is characterised by $\rho_{*} \approx 1 / 450$. Profiles close to experimental ones from Tore Supra have been used, in particular $\nu_{*}$ that weighs the normalised collision term Fig. 1, the safety factor $q$, the density profile, which is constant in time (given the assumption of adiabatic electrons), and the initial ion temperature profile. The safety factor, close to one towards the core increases monotonically to $r / a=1$ where it reaches a relatively large value, $q_{a} \approx 4$. The ion-ion collision frequency $\nu_{i, i}$ is determined by:

$$
\nu_{i, i}=\frac{4 \sqrt{\pi}}{3}\left(\frac{q_{i}^{2}}{4 \pi \epsilon_{0}}\right)^{2} \log (\Lambda) \frac{n v_{t h i}}{T_{i}^{2}}
$$

where $\epsilon_{0}$ is the free space permittivity, $\log (\Lambda) \approx 17$ is the Coulomb logarithm and $v_{t h i}^{2}=T_{i} / m_{i}$ is the ion thermal velocity. The dimensionless control parameter for the collisionality is defined as $\nu_{*}=q R_{0} \nu_{i, i} /\left(v_{t h i} \varepsilon^{3 / 2}\right)$ :

$$
\nu_{*}=\frac{4 \sqrt{\pi}}{3}\left(\frac{q_{i}^{2}}{4 \pi \epsilon_{0}}\right)^{2} \log (\Lambda) \frac{q R_{0}}{\varepsilon^{3 / 2}} \frac{n}{T_{i}^{2}}
$$

In this expression $\varepsilon$ is the inverse aspect ratio: $\varepsilon=r / R_{0}$. The collisionality profile can then be determined, Fig. 1. The collisionaliy is rather large with values from 0.2 to 0.4 in the inner simulation region and a decade increase towards the outer boundary. In the following, we mainly analyse the dynamics of the plasma considering the normalised electric potential $\phi, \phi=e U / T_{0}$.

To complete the simulation characteristics the ion heating source profile and the radial diffusion profiles in the 


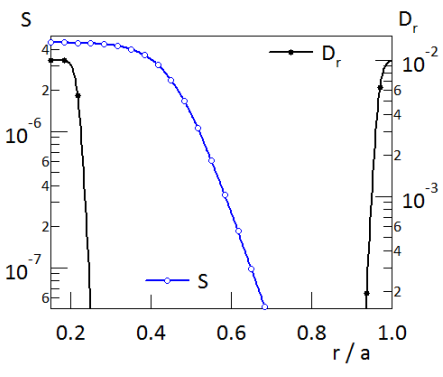

Fig. 2. Profiles used in the simulation, left hand side heating source $S$ and radial diffusion coefficient in the buffer regions $D_{r}$.

Table 1. Main parameters of the $\rho_{*}=1 / 300$ simulation, profiles from Tore Supra shot TS45511, GYSELA SVN version 606 . Torus indicates the fraction of the torus actually simulated.

\begin{tabular}{llllll}
\hline$\Omega_{0} t_{\text {step }}$ & $\Omega_{0} t_{\text {diag }}$ & $N_{\psi}$ & $N_{\theta}$ & $N_{\varphi}$ & Torus \\
\hline 20 & 240 & 512 & 512 & 128 & $1 / 4$ \\
\hline $1 / \rho_{*}$ & $R_{0} / a$ & $r_{\text {int }} / a$ & $r_{\text {ext }} / a$ & $T_{i} / T_{e}$ & \\
\hline 300 & 3.3 & 0.15 & 1.0 & 1 & \\
\hline
\end{tabular}

buffer regions is shown on Fig. 2. The buffer regions are localised at the inner and the outer radial boundaries. The source term is broad, typically from $r / a=0.15$ to $r / a \approx 0.4$.

Two simulations are used is this paper with different $\rho_{*}$ values, $1 / \rho_{*}=150$ and $1 / \rho_{*}=300$. In both simulations, the same set of experimental data is used. Only the major radius $R_{0}$ and the minor radius $a$ are changed at fixed aspect ration $A=R_{0} / a$. As a consequence, the growth rate of the ITG mode of the order of $1 / \tau=c_{0} /\left(q R_{0}\right)=$ $c_{0} /(a q A)$ is also changed with $\tau_{300} / \tau_{150}=2$. However, $\tau_{\|} c_{0} / a=q A$ is unchanged. For the mid-radius safety factor $q \approx 1.6$ one obtains $\tau_{\|}=5.3\left(a / c_{0}\right)$

\section{Interplay between large and small scales of the electric potential}

\subsection{Large scale flow pattern}

In this Section we use data from a $\rho_{*}=1 / 300$ case. Simulation parameters are summarised in table 1 , profiles are presented in Section 3.

We concentrate here on data from a poloidal plane, $(r, \theta)$ plane, at $\varphi=0$. We can then have information on poloidal modes and their radial structure. Note that these correspond to modes in terms of the geometrical angle and not of a magnetic angle. Moreover, this data does not allow one to discriminate between the toroidal mode numbers. Despite these drawbacks, the complex pattern in time and radius of the $\boldsymbol{E} \times \boldsymbol{B}$ flow allows one investigating the intricate self-organised interplay between modes at different scales. Regarding the specific $m=0$ mode, we have
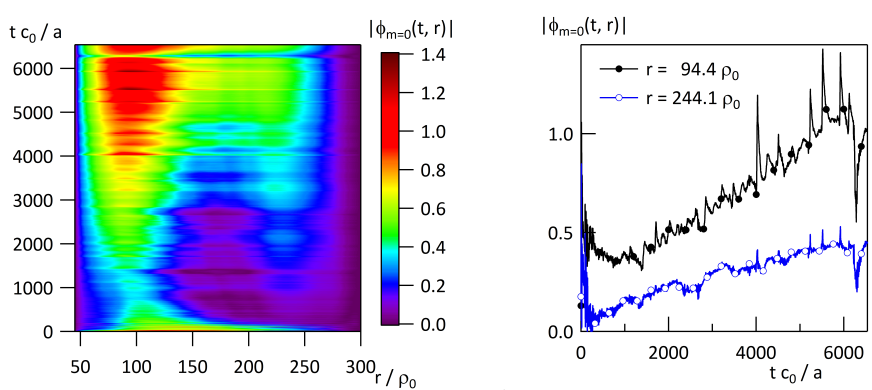

Fig. 3. Contour of the $m=0$ mode of the electrostatic potential $\phi$, left hand side: evolution of the radial profile, right hand side: time trace at two given radial locations.

checked when the data is available that $\left\langle E_{r}(\theta, \varphi=0)\right\rangle_{\theta}$ averaged over $\theta$ hence the $m=0$ mode of the radial electric field is nearly identical to $\left\langle E_{r}(\theta, \varphi)\right\rangle_{f s}$, namely the flux surface averaged radial electric field. On the basis of this result we shall consider in the following that the $m=0$ mode at given $\varphi$ can be regarded as a convenient proxy for the actual zonal flow.

Let us first consider the small mode numbers, in particular $m=0$ that yields $\langle\phi\rangle_{\theta}$, Fig. 3. One finds that this mode is global hence with large scale variation both in time and radially, Fig. 3 left hand side. The radial variation determines the $\boldsymbol{E} \times \boldsymbol{B}$ flow in the poloidal direction. The time-trace at two radial locations, Fig. 3 right hand side, indicates that the simulation has not reached steady state conditions since the amplitude of the mode is increasing linearly.

One also notices relaxation events, either a burst with a rapid increase of the $m=0$ mode amplitude followed by a slower decay or the inverse pattern with a sharp drop of the amplitude and a slower recovery. The amplitude of these bursts appears to increase over most of the profile from the outer towards the inner radius. In order to investigate the radial structure of this burst we determine the local minima and maxima of the time traces at each radial position, which allows one to determine the rise time of the burst. For the large event in the vicinity of time $4000 a / c_{0}$ one finds that the rise time from the local minimum of the time trace to the local maximum is typically of $9.6 a / c_{0}$ with a small radial variation. This value is comparable to the magnitude of the characteristic ITG growth time, $\tau_{\|}$, see Section 3.

For the chosen burst, one finds that the local minimum of the mode amplitude prior to the burst occurs at the same time at all radial positions within the uncertainty of the resolution time of the diagnostic, $\leq \tau_{\text {diag }}=$ $240 \rho_{*} a / c_{0}=0.8 a / c_{0}$. The local maximum of the time trace is then first reached for the time trace at $r=224 \rho_{0}$. Towards the inner and outer radius compared to $r=$ $224 \rho_{0}$, one observes a delay to reach the maximum. At $r=124 \rho_{0}$ this time delay is $1.2 a / c_{0}$. For $r<124 \rho_{0}$ the burst structure is difficult to identify. Towards the outer radius, the maximum is reached with a delay of $2.8 \mathrm{a} / \mathrm{c}_{0}$ at $r=294 \rho_{0}$. The maximum of the pulse thus appears 

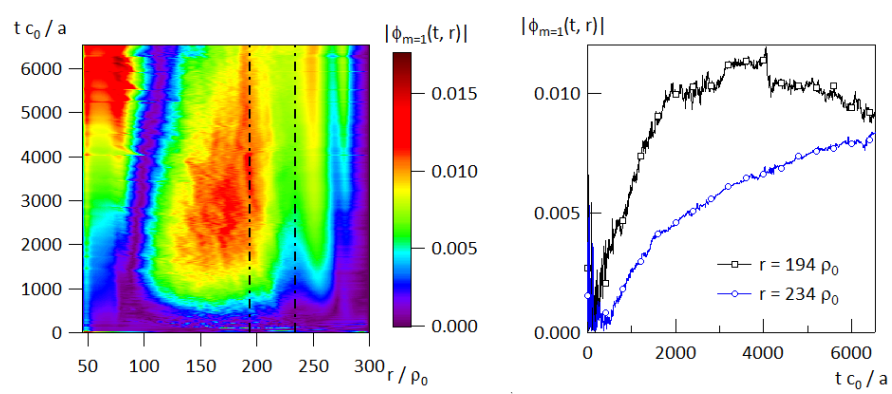

Fig. 4. Contour of the $m=1$ mode of the electrostatic potential $\phi$, left hand side: evolution of the radial profile, right hand side: time trace at two given radial locations.

to originate from $r=224 \rho_{0}$ and to propagate inward at velocity $53 \rho_{*} c_{0}$ and outward at velocity $20 \rho_{*} c_{0}$. These velocities appear to be rather high compared to the drift velocity magnitude $\left(\rho_{*} c_{0}\right)$ and thus appear to reflect some global response of the mode structure. This behaviour is reminiscent of the "gong-mode" [21, 22], an MHD event occurring in conjunction to sawteeth, although characteristics of the modes seem to be altogether different.

It is to be noted that such an analysis applied to the local minimum would yield a propagation velocity of the order of $c_{0}$, namely the propagation over the whole minor radius over a time comparable to the diagnostic time scale, $\approx a / c_{0}$. However, the minimum is very soft and therefore difficult to relate to the burst occurrence. A trigger remains to be determined for such bursts. As noticeable on the time traces, Fig. 3 right hand side, these do not appear to govern a strong change in the overall evolution of the mode.

The $m=1$ mode will likely exhibit toroidal variation that cannot be addressed here. However, except for the $n=0$ toroidal mode none of these modes will have specific radial localisation due to resonances. In that respect it is similar to the $m=0$ mode. However, its analysis yields rather different results. First the mode amplitude is two orders of magnitude smaller. Second, see Fig. 4 left hand side, one finds a zero line of the amplitude that splits the profile of the mode in two regions. This indicates that the radial mode structure is dipolar unlike the $m=0$ mode. As for $m=0$, the mode structure is global. The time traces, Fig. 4 right hand side, appear to be closer to steady state and do not exhibit large bursts. Finally, one can notice structures that are localised in the radial direction with meso-scale duration.

The analysis of the two global modes $m=0$ and $m=1$ allows one to determine the large scale flows. Given the amplitude of the two modes $\phi_{m=0}$ and $\phi_{m=1}$ and their similar radial scales, one finds that the main large scale poloidal velocity component is $\left\langle v_{E \theta}\right\rangle=\rho_{0} \partial_{r} \phi_{m=0}$, where the average is a poloidal average. As explained above, we will consider this velocity as a proxy for the zonal flow $v_{Z}$. The latter is found to exhibit a rather complex amplitude and sign reversal pattern [23], see Fig. 5. The change
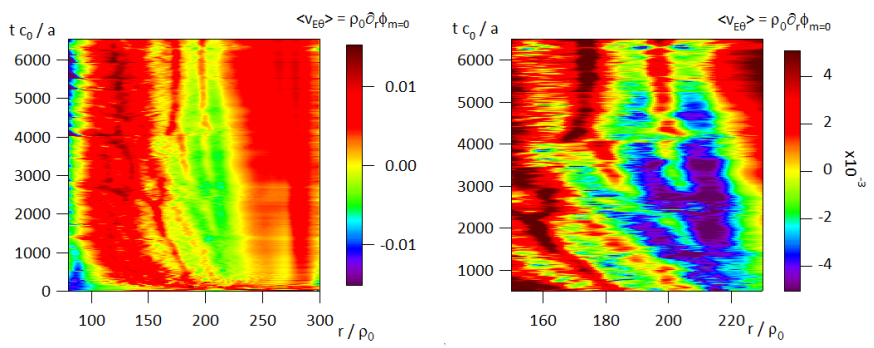

Fig. 5. Zonal flow contour of $\left\langle v_{E \theta}\right\rangle \mid$ : left hand side, and zoom of $\left\langle v_{E \theta}\right\rangle$ : right hand side.
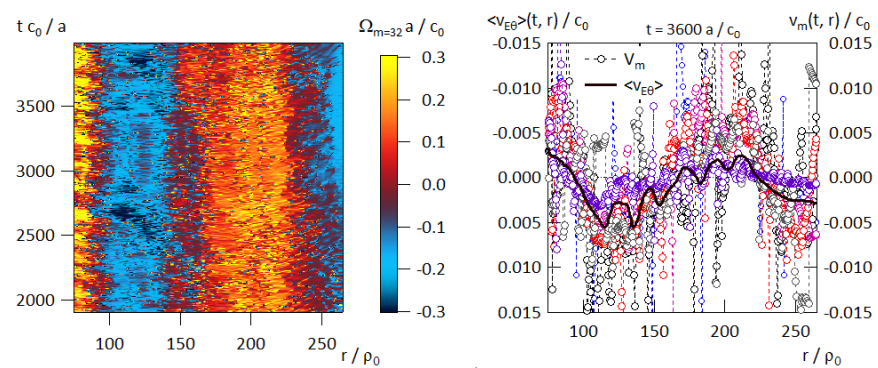

Fig. 6. Contour of the pulsation $\Omega_{m}(t, r)$ of the $m=32$ mode of the electrostatic potential $\phi$ : left hand side, profile of the zonal flow $v_{Z}=\left\langle v_{E \theta}\right\rangle$, plain black line, and mode velocity of several modes $v_{m}(t, r)=\Omega_{m}(t, r) / k_{m}$ : right hand side, $m=16$ black dashed lines and circles, $m=24$ dark blue dashed lines and circles, $m=32$ magenta dashed lines and circles, $m=36$ blue dashed lines and circles, $m=64$ red dashed lines and circles, $m=128$ black dashed lines and diamonds.

in amplitude, with local minima and maxima will govern shearing effects, see Section 6 , while the change in sign will lead to changes of the sign of the slope of apparent dispersion relations, a feature that is outstanding from that perspective, see Section 4.2.

For this simulation, one finds zonal flow reversal at several radial locations. However, on the overall, the radial profile evolves slowly and the flow pattern is characterised by a negative flow at the inner boundary, followed by a rather sharp transition to a positive flow. Around midradius a more complex pattern is observed, Fig. 5 right hand side, mainly with negative flow. Finally towards the outer radius there is a large region with positive flow.

\subsection{Apparent dispersion relation}

One can also observe the modulation of the mode amplitude in time that is readily associated with oscillations of the phase of the mode. Reconstructing the latter and analysing its dependence on time, one can determine the pulsation of each mode, $\Omega_{m}(t, r)$. As an example a contour plot of $\Omega_{32}(t, r)$ is shown on Fig. 6: left hand side.

Given the pulsation of the various modes, one can readily determine the phase velocity of the modes defined by $v_{m}(t, r)=\Omega_{m}(t, r) / k_{m}$ where the wave vector is defined 
as $k_{m}=m / a$. One finds that these phase velocities have the same order of magnitude and exhibit the same large scale features regarding their radial profiles, see Fig. 6: right hand side. It si important to stress that the phase velocity is thus quite comparable for mode numbers ranging from 16 to 128. Furthermore, the radial profile of the zonal flow velocity $v_{Z}$ is found to compare reasonably well with the phase velocity of the modes, Fig. 6: right hand side. The correlation between the zonal flow and the phase velocity $v_{m}(t, r)$, as exemplified on Fig. 6 : right hand side, is not a one to one relation. Indeed, only the major trend is recovered and at smaller scales, significant departure between the phase velocities of the various modes and the zonal flow is observed.

In particular, one readily notices that the profile as well as the trace of $v_{m}(t, r)$ exhibit strong peaks. These appear to be actually governed by sudden acceleration and deceleration of the phase of the modes, an effect that is under investigation. The difference between the phase velocity and the zonal flow changes sign along the radial profile, Fig. 6: right hand side, and is not therefore governed by as a simple shift due to a constant velocity difference.

A robust feature in the analysis appears to be the linear dependence of the mode pulsation $\Omega_{m}(t, r)$ on the mode number $m$, Fig. 7 . This leads to a presentation of the data in the form of a pseudo-dispersion relation as has been reported in KSTAR experiments [24]. For the sake of comparison we introduce the apparent pulsation $\Omega_{E m}=k_{m}<v_{E \theta}>$ due to the Doppler shift governed by the zonal flow. One finds that in both cases shown on Fig. 7, the pulsation associated to the Doppler shift tends to overestimate the actual mode pulsation. However, this situation is not generic and at other times and radial positions one can obtain an underestimate Fig. 6: right hand side. Consequently, it is difficult to relate the difference between these pulsations, namely the intrinsic mode pulsation, to the density diamagnetic frequency [5] as found in the linear analysis [5].

An important output of the analysis in terms of a dispersion relation, as done in Fig. 7, is to provide in fact an estimate of the zonal flow, resolved in time and radial position. The striking aspect reported in the experimental investigation, namely the reversal of the slope of the apparent dispersion relation, would then indicate a reversal of the zonal flow.

\section{Structure of the electric potential along the field line}

In this Section we analyse the $\rho_{*}=1 / 150$ case. The simulation parameters are summarised in table 2 .

The time trace of the maximum and minimum fluctuations of the electrostatic potential $\delta \phi$ at a given radius are shown on Fig. 8. These exhibit first a sharp rise corresponding to the linear growth stage of the ITG instability, from $t c_{0} / a \approx 60$ up to $t c_{0} / a \approx 80$. Following the first burst of turbulent activity, the system undergoes a cyclic
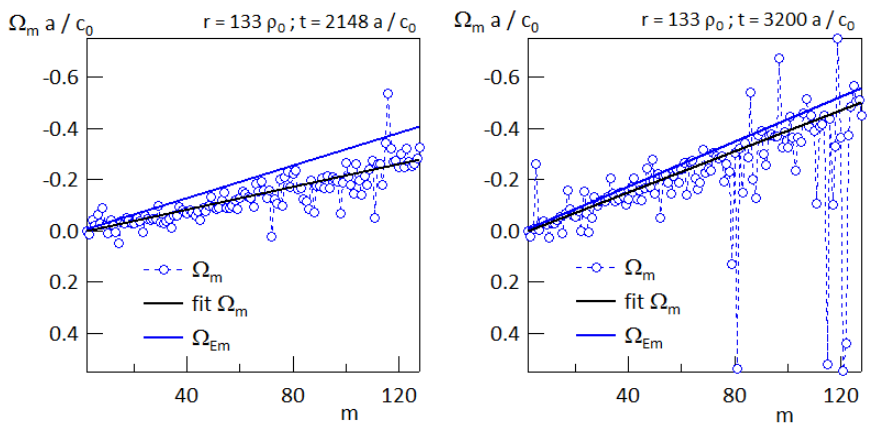

Fig. 7. Dispersion-like relationship between the mode pulsation $\Omega_{m}(t, r)$ and the mode number $m$ at a given radial location $r=135 \rho_{0}$, and time, $t=2148 a / c_{0}$; left hand side, and $t=3200 a / c_{0}$.

Table 2. Main parameters of the $\rho_{*}=1 / 150$ simulation, profiles from Tore Supra shot TS45511, GYSELA SVN version 606

\begin{tabular}{llllll}
\hline$\Omega_{0} t_{\text {step }}$ & $\Omega_{0} t_{\text {diag }}$ & $N_{\psi}$ & $N_{\theta}$ & $N_{\varphi}$ & Torus \\
\hline 20 & 180 & 256 & 256 & 128 & $1 / 2$ \\
\hline $1 / \rho_{*}$ & $R_{0} / a$ & $r_{\text {int }} / a$ & $r_{\text {ext }} / a$ & $T_{i} / T_{e}$ & \\
\hline 150 & 3.3 & 0.15 & 1.0 & 1 & \\
\hline
\end{tabular}
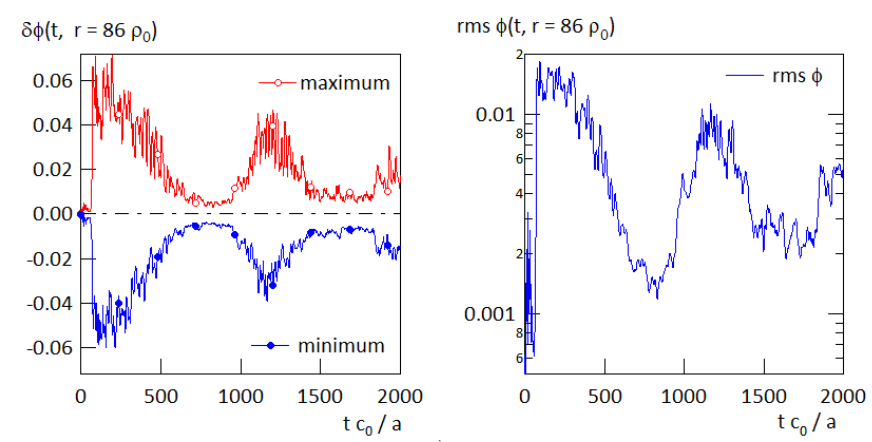

Fig. 8. Left hand side trace: maximum and minimum of the electrostatic potential $\phi$, right hand side trace: r.m.s. of the fluctuations of the electrostatic potential.

behaviour with alternate periods of strong and weak turbulence activity.

These features are readily recovered when analysing the time trace of the root mean square (r.m.s) of the electrostatic potential fluctuations Fig. 8. After a transient time during which the initial perturbation reorganises according to its projection on the stable and unstable modes, the instability is found to grow exponentially with a characteristic time scale of $3.2 a / c_{s}$. The latter can be compared to the typical parallel transit time, $\tau_{\|}=$ $\left(q R_{0} / a\right) a / c_{s}$ that governs the reversal of the curvature drift, and therefore the charge separation generating the electrostatic potential of ITG modes. For $q \approx 1.6$ and the given value of $R_{0} / a$, table 2 , one obtains $\tau_{\|} \approx 5.3 \mathrm{a} / \mathrm{c}_{\mathrm{s}}$. 


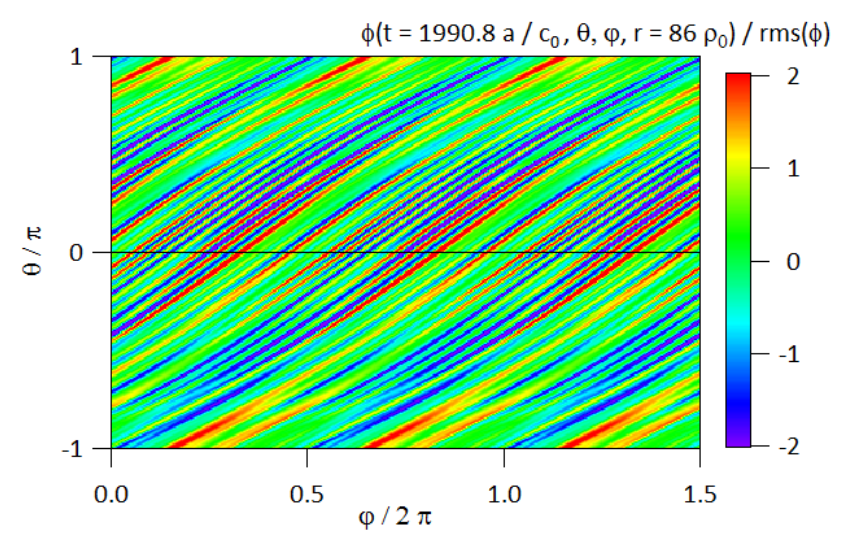

Fig. 9. Magnitude of the electrostatic potential in the plane of a magnetic surface $(\theta, \phi)$. The potential is normalised by its root mean square $(\mathrm{rms})$. The computed data on a half torus is duplicated $3 \approx 2 q$ times in the toroidal direction, hence taking account of the actual magnetic geometry, to give a clearer picture of the parallel structure of the filaments.

The order of magnitude of the observed rise time of the electrostatic fluctuations corresponds therefore to that of the transit time $\tau_{\|}$. Conversely, the subsequent modulations are much slower, $645 a / c_{s}$ for the decay of the first turbulence burst, $760 a / c_{s}$ for the rise an decay time of the first modulation. An intermediate time scale is thus found to govern the behaviour of the turbulence when the system is weakly driven out of equilibrium.

\subsection{Electric potential structure on a magnetic surface}

The runs of full-f and global gyrokinetic codes generate too much data to store all the outputs. In practice, only a small fraction of the information is available for analysis. The flux surface average of the heat flux exhibits an intermittent like behaviour with ballistic transport events. These events have the same signature as that observed in fluid codes that we refer to as avalanches. In a loose way, these are reminiscent of SOC avalanches [11, 15]. By considering the $(\theta, \varphi)$ plane at mid radius, $r=86 \rho_{0}$, we investigate how localised can these avalanches be in the toroidal direction. Indeed, the latter have been identified in fluid simulations, but assuming a flute mode symmetry $[8,25]$, as well as in gyrokinetic simulations $[5,15,26]$, but generally considering flux surface averaged quantities. A critical issue in this process is to identify within the evolving structure of the electric potential, events that are effectively correlated to avalanches. To that end, we assume that the largest deviation of the electric potential in terms of rms are associated to large avalanches that puncture the given magnetic surface. A threshold at twice the $r m s$ value is used here to detect the avalanche dynamics in the electric potential on a given magnetic surface.

The 2D plot of the normalised electrostatic potential, Fig. 9, allows one to analyse its structure. First one finds that it tends to a dipolar structure with filaments alter-
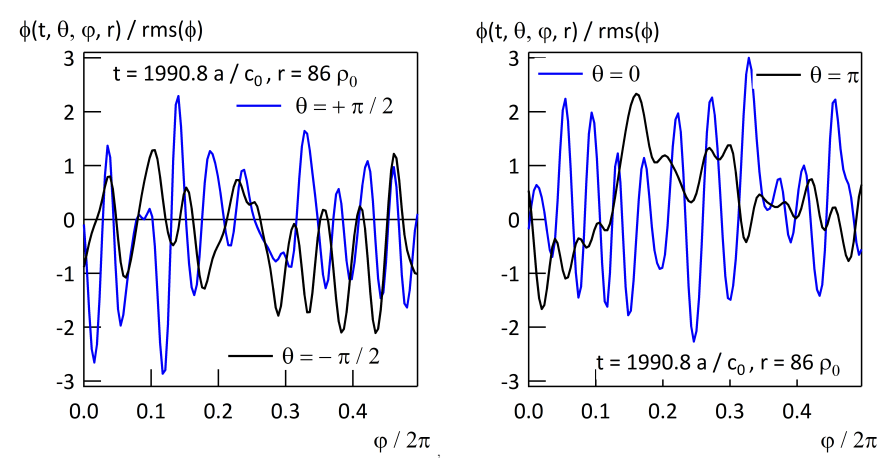

Fig. 10. Successive snapshots of the electric potential drawn along the line parallel to the maxima of the bursts, left hand side decay of a burst, right hand side growth of another burst.

nating signs. These filaments are in first approximation aligned on the field lines with parallel extent typically of order $1 / \rho_{*}$ compared to the transverse scales in agreement with the drift-scaling. They are found to exhibit a finite parallel extent, hence with clear departure from flute structures characterised by $k_{\|}=0$. Note that at $r=86 \rho_{0}, q \approx 1.6$ so that 1.5 turns toroidally are necessary for one turn in $\theta$, as readily observed on Fig. 9. While the magnetic equilibrium and the drive of ITG turbulence is up-down symmetric, one finds that at the time of the snapshot there is loss of up-down symmetry. Indeed, the dipolar structure with minima and maxima departing by typically $1 \mathrm{rms}$ are localised above $\theta=0$, hence the low field midplane. Below the low field mid plane the structures have smaller negative amplitude and near zero positive amplitude, see Fig. 10 left hand side. This symmetry loss appears to be related to zonal flows. This point will be addressed in Section 6. Comparing the low field $\theta=0$ and the high field midplane $\theta=\pi$, Fig. 10 right hand side, the dipolar structures are smoothed out leading to a structure with longer toroidal wave length.

\subsection{Expansion and retraction of the electric potential burst}

With a threshold method we then identify the events such that $\phi / r m s(\phi) \geq 2$. We present here the evolution, time step after time step, of the profile of $\phi$ interpolated along the line of maximum amplitude of the structure. The lack of resolution in the $\theta$-direction introduces some short scale sawtoothing that is irrelevant (the localisation of the maximum amplitude is not interpolated). Furthermore, this poor resolution does not allow one to easily determine a departure between the field line direction and the line of maximum amplitude of the electric potential. However, this method allows one to follow the growth and decay of a large structure (strong deviation in terms of $\mathrm{rms}$ ) and in particular analyse its expansion in the parallel direction Fig. 11. For the sake of simplicity in this representation, the toroidal angle $\varphi$ is used as curvilinear abscissa along the field line. 

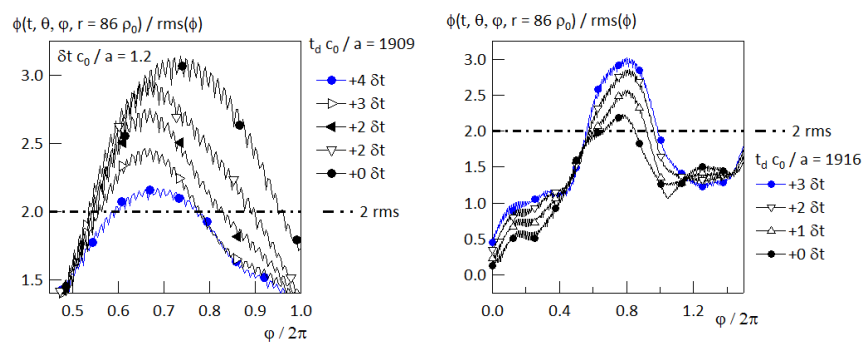

Fig. 11. Variation of $\phi / r m s(\phi)$ along a filament (the curvilinear abscissa is labelled by $\varphi$ ), left hand side during the decay phase of a burst, right hand side during the expansion of another burst.

On the left hand side of Fig. 11 are plotted successive parallel profiles of the electric potential during the decay of such a burst. One finds that the characteristic time scale of these bust events is of the order of $5.4 a / c_{0}$, hence comparable to $\tau_{\|}$. The structure is observed to retract mainly from the right hand side $\varphi /(2 \pi) \approx 0.955$ at $\delta t=0$ towards the left hand side (decreasing values of $\varphi), \varphi /(2 \pi) \approx 0.775$ at $\delta t=3 a / c_{0}$ the point at $\varphi \approx \pi, \phi / r m s(\phi) \approx 1.5$ being approximately a fixed point. Neglecting the poloidal component of the velocity with respect to the toroidal component (error of the order of $\left.0.5\left(q * R_{0} / a\right)^{2}\right)$, one thus finds that the front retraction velocity is $\approx 1.04 c_{0}$.

Performing a similar analysis for the growth of another burst, Fig. 11 right hand side, one finds that it lasts $\approx 4.2 \mathrm{a} / \mathrm{c}_{0}$, the expansion being near symmetric towards the positive and negative values of $\varphi$ and starting from $\approx 0.8(2 \pi)$ where the electric potential increases above the threshold value. One finds that the maximum expansion of the burst along $\varphi$ reaches $0.86(2 \pi)$, hence yielding a front velocity of $\approx 1.06 c_{0}$. Note that one can also observe a fixed point during this growth at $\varphi \approx \pi$ and $\phi / r m s(\phi) \approx 1.7$.

For the two structures, one finds that the maximum extent along $\varphi$ is $\delta \varphi \approx 0.8 \pi$ which corresponds to $\delta \theta=$ $\delta \varphi / q \approx 0.5 \pi$ hence $\pm 45^{\circ}$ with respect to the midplane. This is in relatively close agreement with the analysis of the ballooned structure of the ion heat flux analysed in GYSELA [26]. Interestingly this value also matches experimental results for the SOL transport in L-mode [27-29].

Although it appears reasonable to consider that such bursts result from an avalanche breaking through a magnetic surface at a localised toroidal position and then expanding along the field line to create a filamentary structure, one must still assess the correlation of such bursts of the electric potential structure with that of the heat flux. A 3D analysis is also necessary to discriminate between such a mechanism and radial motions of filaments with constant extent and bulging across the magnetic surface.

\section{Interplay between zonal flow shear layers and avalanche heat transport}

In this Section we use data from the $\rho_{*}=1 / 300$ case. Simulation parameters are summarised in table 1 , profiles are presented in Section 3.

The target of this section is to identify avalanches $[6,8,13,25]$ and investigate their interplay with the corrugation structures $[14,16,30]$. As already underlined, the output from the large gyrokinetic simulation is not complete. Our present analysis is mainly restricted to the dynamics in the poloidal plane at $\varphi=0$.

In the study of the self organised transport, they are several aspects to take in account,(i) the identification of structures or patterns, and consequently a first analysis of the underlying process that governs the self organisation of the system, (ii) the evolution and propagation in space of the structures, (iii) the mechanisms driving relaxation events and (iv) the interplay between the various structures.

We focus our attention on the latter aspect. In fact, since the corrugations (also called staircases) are defined as micro-barriers combining organised shear layers and a local increase of the temperature gradient [16], a straightforward issue is then to analyse the response of avalanches, namely the most efficient radial transport mechanism, to the micro-barrier. An interesting issue is to understand why some avalanches are able to destroy the micro-barrier while others are just dumped away? And consequently, is there evidence of a threshold effect that governs the disruption of micro-barriers and therefore level off their favourable impact on confinement?

\subsection{Zonal flow shear layers}

In Section 4, the zonal flow pattern of the simulation $1 / \rho_{*}=300$ is investigated in detail. The radial profile of the zonal flows is characterised by a series of minima and maxima and includes flow reversal [9]. This flow pattern determines the regions where the shear $\left|\rho_{0} \partial_{r} v_{z}\right|$ is strong and can modify the radial transport properties [10]. On Fig. 12 left hand side, one can observe shear regions where the zonal flow reverses. One can also see that the large shear values are not restricted to these specific locations, Fig. 12 left hand side (note that only the shear values larger than 0.25 are plotted). In the mid-radius region, one can notice that the shear maxima generate patterns, as mentioned in $[9,25,31]$. In particular, one can notice pairs of high zonal flow shear regions, localised radially and evolving on meso-scale times Fig. 12 right hand side.

These form a structure of small barriers that have been shown to govern a local increase of the radial temperature gradient, so called corrugations, and leading to a stair case like temperature profile [17]. For the sake of convenience, we shall refer to such a pattern as a corrugation. A close-up view of such a structure is plotted on Fig. 13, left hand side. One can readily notice that the corrugation pattern shifts radially (while the safety factor profile is fixed) and exhibits modulations at high frequency and even disruptions, namely transient loss of the pattern before it re-appears in a neighbouring radial location Fig. 13, left hand side. 

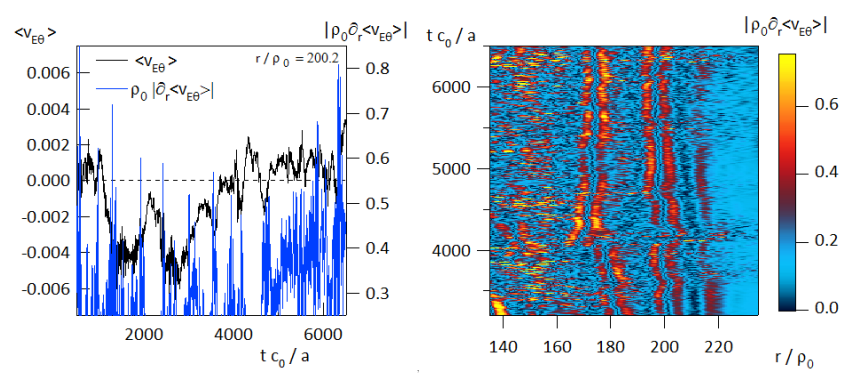

Fig. 12. Time trace of the zonal flow magnitude $\left\langle v_{E \theta}\right\rangle$ (left hand scale) at $r / \rho_{0}=200.2$ and zonal flow shear $\left|\rho_{0} \partial_{r}\left\langle v_{E \theta}\right\rangle\right|$ at the same location (right hand scale) : right hand side. Contour of the zonal flow shear $\left|\rho_{0} \partial_{r}\left\langle v_{E \theta}\right\rangle\right|$ : right hand side.
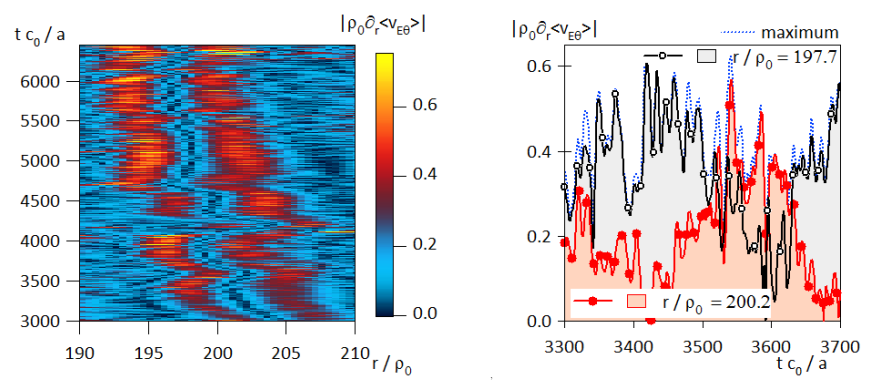

Fig. 13. Contour of zonal flow shear $\left|\rho_{0} \partial_{r}\left\langle v_{E \theta}\right\rangle\right|$ zoomed in a region with shear layers: left hand side and evolution of the zonal flow shear; dashed blue line maximum for $197.2 \leq$ $r / \rho \leq 200.7$, black line, open symbols value for $r / \rho=197.7$ red line open symbols for $r / \rho=200.2$ : right hand side.

Evidence of this behaviour is found on Fig. 13, right hand side, where the maximum of the zonal flow shear in a radial window closely matches the maximum of the zonal flow shear from two different radial coordinates. This is consistent with a movement of the pattern between these two neighbouring radial locations during the chosen time window. One can also clearly notice on these time traces that the corrugation exhibits several time scales in its evolution pattern, the radial displacement corresponding to both rapid steps on short time scales and gradual shift on the long time scales. Scale separation that would allow one to proceed to time averages can then only be achieved on the basis of an efficient pattern recognition process (which is beyond the scope of this analysis).

When increasing the poloidal mode number from $m=$ 0 , which yields a poloidal flow comparable to the zonal flow, to $m=2$ and higher mode numbers, one can observe two features. For $m=1$ and $m=2$ the mode structure exhibits a correlation with the large shear regions of the zonal flows, Fig. 14: left hand side. This pattern is also characterised by a slow evolution in contrast to the higher mode numbers. Beyond $m=2$, the mode amplitude pattern is characterised by higher frequency events and rather homogeneous radial profiles, see Fig. 14: right hand side.

On the latter figure, the black dots correspond to local maxima of the zonal flow shear. One can observe that

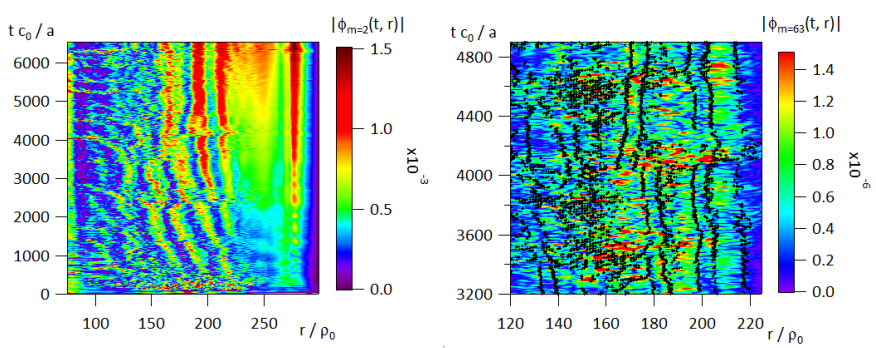

Fig. 14. Contour of the amplitude of the $m=2$ mode of the electrostatic potential $\phi$ : left hand side, and trace of the profile of amplitude of the $m=63$ mode: right hand side. The points with high zonal flow shear are marked by a black dot. This provides a means to localise the corrugations.

they are two regimes that exhibit a strong correlation between these dots and the large amplitude of the $m=63$ mode. When the dots exhibit the long lived corrugation structure, they appear to bound regions with rather homogeneous amplitude of the $m=63$ mode. Conversely, when the dots are more oriented along outward ballistic trajectories, they can be localised at the same location as the maxima of the $m=63$ mode amplitude. It seems therefore that the shearing effect tend to confine the fluctuations in the case of the corrugation pattern, and, when such a pattern does not exist, can be transported with a ballistic motion comparable to that of an avalanche.

\subsection{Definition of micro-barriers}

Determining the effect of the transport barrier on the temperature field $T_{i}$ is not straightforward since the latter is evolving throughout the simulation. This is readily observed on Fig. 15 left hand side. In the radial interval of interest one finds that the temperature increases by $50 \%$. Although the variation during the time window used in the following, typically from $t c_{0} / a=3600$ to $t c_{0} / a=4100$ appears to be small, Fig. 15 left hand side, it is still large compared to the fluctuation level. It is easier to consider the heat flux $Q$ [15, 20, 26], Fig. 15 right hand side. Both the temperature field and the heat flux that are considered in this Section are averaged on a magnetic surface. Localised effects on a surface are then levelled off.

Let us compare the total heat flux through a magnetic surface $r Q_{\text {tot }}$ to its neoclassical contribution $r Q_{\text {neo }}$ and the turbulent one $r Q_{\text {turb }}$. In the radial window of interest, one finds that the total heat flux is approximately constant while both the neoclassical and turbulent heat flux exhibit oscillations that compensate each other when computing the total flux. One thus finds that when the turbulent flux is depressed, the neoclassical flux increases. When analysing such a behaviour in the framework of diffusive transport, one finds:

$$
<Q_{t o t}>=-n\left(\alpha \chi_{t u r b}+(1-\alpha) \chi_{n e o}\right) \partial_{r}<T>
$$

where $\alpha$ is the fraction of the heat flux due to turbulent transport. In such an expression, ones assumes $\chi_{\text {turb }}$ and 
$\chi_{n e o}$ invariant and given such that $\chi_{\text {turb }} \gg \chi_{n e o}$. In quasi steady state conditions with fixed density, $\left\langle Q_{t o t}>/ n\right.$ is constant and $\left(\alpha \chi_{\text {turb }}+(1-\alpha) \chi_{\text {neo }}\right) \partial_{r}<T>$ is also constant. Let us define the temperature gradients $\nabla_{r} T_{\text {turb }}$ and $\nabla_{r} T_{n e o}$ required respectively for $\alpha=1$ and $\alpha=0$ for this given total flux, one then obtains:

$$
\frac{<Q_{t o t}>}{n}=\chi_{\text {turb }}\left|\nabla_{r} T_{\text {turb }}\right|=\chi_{\text {neo }}\left|\nabla_{r} T_{\text {neo }}\right|
$$

One then has $\left|\nabla_{r} T_{\text {turb }}\right| \ll\left|\nabla_{r} T_{\text {neo }}\right|$ and more generally $\left|\nabla_{r} T_{\text {turb }}\right| \leq\left|\partial_{r} T\right| \leq\left|\nabla_{r} T_{\text {neo }}\right|$. A large temperature gradient is thus associated to a weak turbulence limit $(\alpha \approx 0)$ and conversely a weak temperature gradient is associated to large turbulent transport $(\alpha \approx 1)$. Given $\left\langle Q_{\text {turb }}\right\rangle$ $/ n=-\alpha \chi_{\text {turb }} \partial_{r} T$ one also finds:

$$
\begin{aligned}
\frac{\left\langle Q_{\text {turb }}>\right.}{\left\langle Q_{\text {tot }}>\right.} & =\frac{\alpha \chi_{\text {turb }}}{\alpha \chi_{\text {turb }}+(1-\alpha) \chi_{\text {neo }}} \\
& =\frac{\alpha\left|\nabla_{r} T_{\text {neo }}\right|}{\alpha\left|\nabla_{r} T_{\text {neo }}\right|+(1-\alpha)\left|\nabla_{r} T_{\text {turb }}\right|}
\end{aligned}
$$

Large turbulent transport, $\alpha \approx 1$ then leads to a small temperature gradient and $\left\langle Q_{t u r b}\right\rangle /\left\langle Q_{t o t}\right\rangle \approx 1$ while weak turbulent transport, $\alpha \approx 0$, the case of improved confinement governed by a transport barrier, leads to a large temperature gradient and $\left\langle Q_{\text {turb }}\right\rangle /\left\langle Q_{\text {tot }}\right\rangle \approx 0$

We thus define a transport barrier to be the region, localised radially but extending over the whole flux surface, characterised by a strong drop of the radial turbulent flux [31], namely when:

$$
R_{B}=\frac{Q_{t u r b}}{Q_{t o t}} \leq 0.3
$$

The figure of merit for the barrier $R_{B}$ is more general than the introduction based on diffusive transport and can readily be generalised to a turbulent flux $Q_{\text {turb }}$ governed by thermal convection due to the $E \times B$ drift velocity, Eq. (3a). Then $Q_{t o t}$ is the total radial heat flux (also averaged on a magnetic surface), hence the sum of turbulent and neoclassical heat flux, namely the flux governed by the curvature drift Eq.( $3 \mathrm{~b})$.

In Fig. 16 we can see that the width of the transport barrier is fluctuating in time. Two large turbulent bursts are observed to break through the barrier, the first between time $t c_{0} / a 3500$ and 3700 and the second between 4000 and 4200 . Two radial intervals, one upstream from the barrier, $\Delta r_{u}$, the other downstream $\Delta r_{d}$ are used to compare the radial and poloidal components of the $E \times B$ velocity in order to understand how the avalanches are impacted by the barrier and vice-versa [31].

When comparing the location of the regions where $R_{B} \leq 0.3$, defined as the corrugation structures, and the location of the maxima of the zonal flow shear, Fig. 16, one finds that there is a common trend but not a perfect match. It seems that the corrugation structure includes two shear layers that are located on the side of the regions where $R_{B} \leq 0.3$ (dark regions on Fig. 16). The corrugation thus seems to be more redily associated to a maximum or a minimum of the zonal flow velocity, the latter
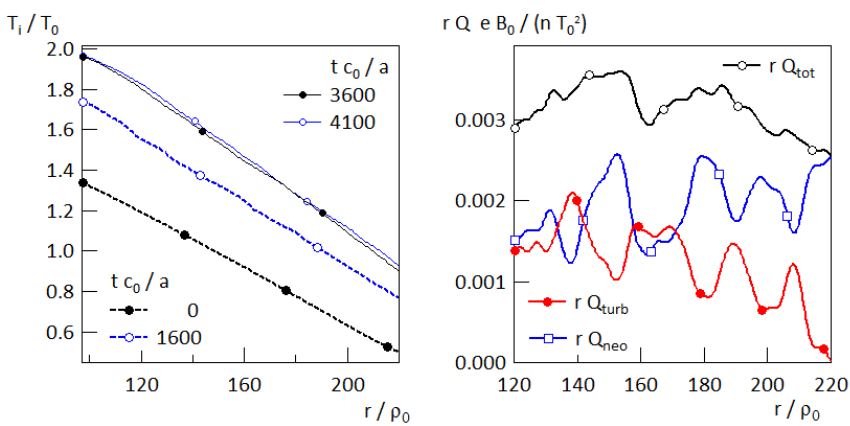

Fig. 15. Ion temperature profiles at different times during the simulation, left hand side and heat flux through a magnetic surface, proportional to $r Q$ for the total (tot), neoclassical (neo) and turbulent (turb) contributions, right hand side.

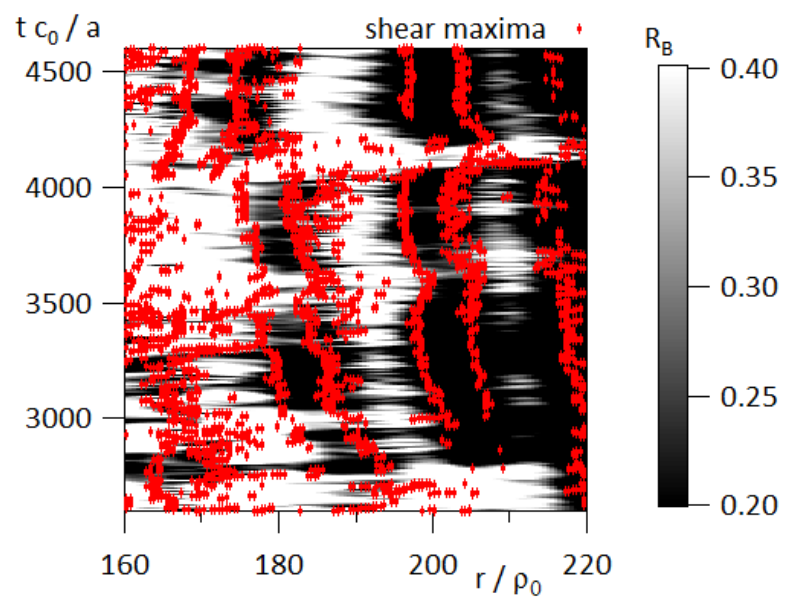

Fig. 16. Correspondence between the barrier criterion $R_{B}$ and the location of the points with maximum zonal flow shear. Left hand side $R_{B}$ is given in scales of grey at $0.3 \pm 0.1,0.3$ being the critical value, see Eq. 14, the points with maximum shear are located by the red dots.
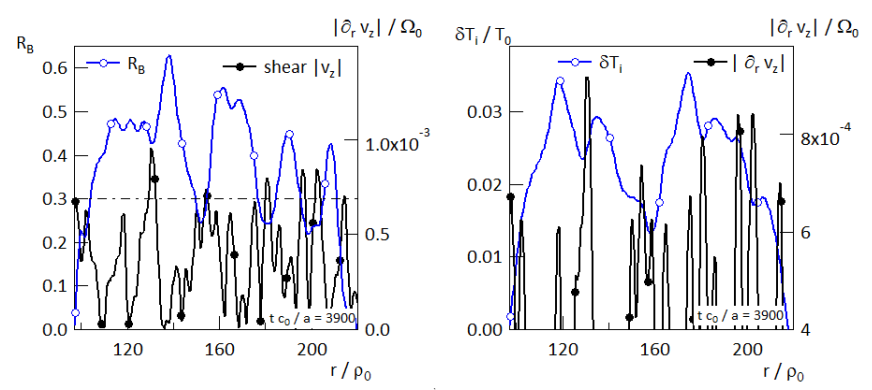

Fig. 17. Left hand side, profiles of $R_{B}$ and $\left|\rho_{0} \partial_{r} v_{z}\right|$ at $t=$ $3600 a / c_{0}$. Right hand side, profile of $\delta T_{i}$ profiles of $\delta T_{i}$, left hand side compared to the zonal flow shear 


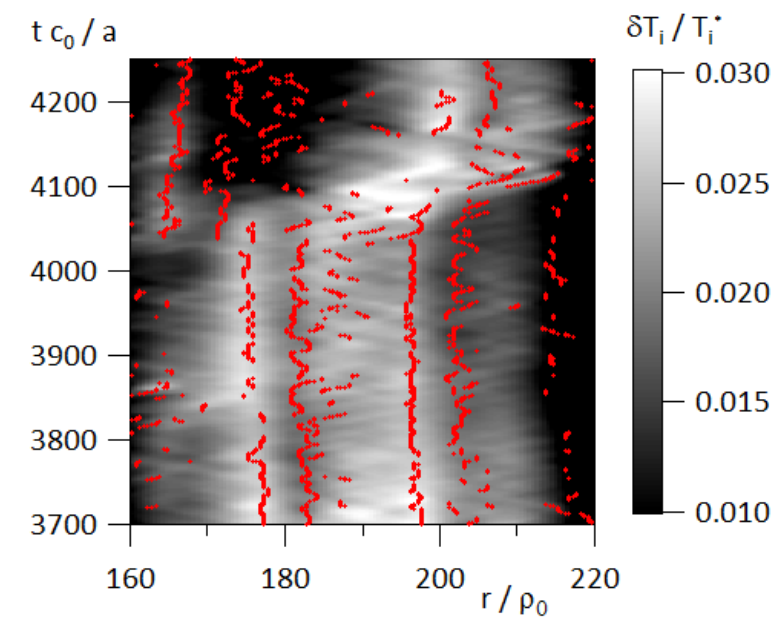

Fig. 18. Variation of $\delta T_{i}$ and superimposed the regions with maximum shear. $\delta T_{i}$ is the difference between the ion temperature and a fit of the variation of the temperature determined as a linear function of the initial profile.

being associated to two regions with zonal flow shear on either side of the zonal flow extremum. However, one can notice the correlation between the corrugation disruption determined by the opening of the regions with maximum zonal flow shear and those where values of $R_{B}>0.3$ exhibit radial ballistic propagation. The profiles at a given time of $R_{B}$ and $\left|\rho_{0} \partial_{r} v_{z}\right|$, Fig. 17 left hand side, underline both the convergence of these indicators in the microbarrier regions as well as differences in their behaviour.

In order to investigate the temperature fluctuations, we have to define a reference temperature without using a mean value since the temperature profile is evolving steadily, and since the fluctuations we want to analyse are small. We fit the profile at time $t c_{0} / a=1600$ as a linear function of the smooth temperature profile used as initial condition. We then use the fact that the temperature profile evolves linearly with time (in a first approximation) to define a reference temperature $T_{r e f}(t, r)$. The temperature variation is then defined as $\delta T_{i}(t, r)=T_{i}(t, r)-T_{r e f}(t, r)$. It includes both the fluctuations and a systematic deviation due to the approximate fit of $T_{r e f}(t, r)$. The evolution of the profile of $\delta T_{i}$ prior and just after time $t c_{0} / a \approx 4100$, Fig. 18, is found to exhibit dynamics at small scale, typically the radial distance between two corrugations of the order of $20 \rho_{0}$ and lasting some $40 a / c 0$, and larger scales and magnitude such as that governing the disruption of the corrugation structure at $t c_{0} / a \approx 4100$, that lasts about $100 a / c 0$ and extends over $40 \rho_{0}$. These events appear to be essentially ballistic with a similar velocity ranging from $0.4 \rho_{*} c_{0}$ to $0.5 \rho_{*} c_{0}$. One can also observe that the small events are constrained by the corrugation structures and appear to bounce back and forth between the shear layers. Regarding the temperature variation $\delta T_{i}$, one can also observe that it tends to peak just prior or at the inner most shear layer of a zonal flow extremum and to be depleted in the vicinity of its outer shear layer, Fig. 18. When analysing the profile of the temperature variation, Fig. 17 right hand side, and comparing it to the profile of the zonal flow shear, including a threshold effect with a non zero scale offset, one finds that the apparent correlation observed on the contour plot is far more difficult to assess.

The various observations of the corrugation structure allow one to draw some general features. First, one must consider that the two shear layers in the vicinity of an extremum of the zonal flow velocity are part of the same structure. These two layers are found to isolate regions of minimum turbulent heat flux and consequently of maximum neoclassical heat flux. The temperature variation appears to reflect this property with peaking prior or at the first shear layer and depletion close to the second shear layer, hence indicating an enhanced gradient between the two shear layers. The difficulty in drawing the correlation between shear layers and transport underlines three aspects of this physics:

- the non linear aspect, the threshold procedure being but one means to highlight it,

- the local features that govern the interaction are levelledoff when considering flux surface averages of the heat flux and temperature field,

- the shear layers that we analyse are not simple objects, they exhibit strong fluctuations in time and space and their continuity even at small time and space scales is questionable,

- the issue of scale separation, which together with geometrical features, is the backbone of separation of the electrostatic potential into shear layers on the one hand and avalanches on the other hand does not seem to hold when addressing their interaction.

\subsection{Trigger mechanism for micro-barrier reorganisation}

Peaks of the temperature variation $\delta T_{i}$ yield a means to investigate the dynamics of the corrugations, Fig. 18 and Fig. 19. One finds that the peaks, such as that at $r \approx$ $145 \rho_{0}$ and time $4023 a / c_{0}$, Fig. 19 left hand side, develop while the points at both radial sides hardly vary. Conversely, the avalanche that coincides with the collapse of the corrugation at time $t c_{0} / a \approx 4100$ clearly exhibits a radial shift of the peak, Fig. 19 right hand side. The analysis of the position of this peak allows one to capture the inward displacement prior to a reflection and the large outward ballistic motion. Following the position of the maximum in time then provides the velocity of this avalanche which is found to agree with the value given previously $0.5 \rho_{*} c_{0}$.

This two-fold description of the evolution of the temperature variation could be related to two different heat transport mechanisms. On the one hand, the avalanche associated to the disruption of the corrugation could exhibit a flux surface symmetry comparable to that of the corrugation. The flux averaged transport event would then appear as being large. On the other hand, the local peaking 

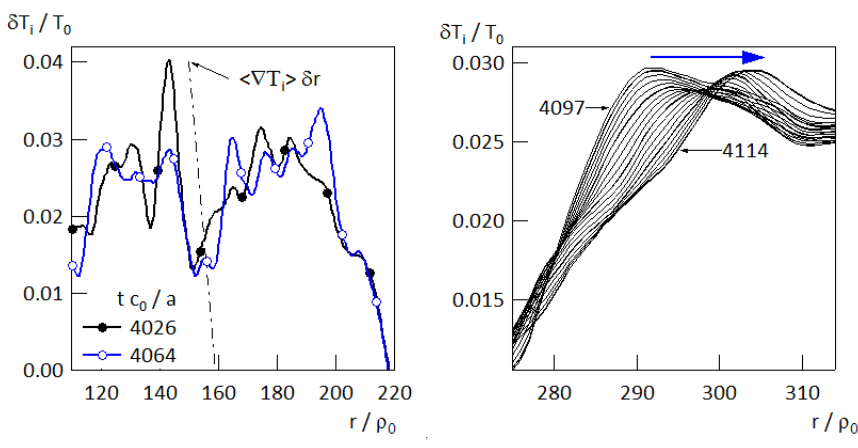

Fig. 19. Variation $\delta T_{i}$ of the ion temperature; left hand side, profiles of $\delta T_{i}$ at two characteristic times and comparison the gradient of the reference temperature at that time, right hand side zoom of the profiles of $\delta T_{i}$ during the propagation of the avalanche associated to the disruption of a corrugation structure.

could be related to the stopping capability of the corrugation of avalanches that are localised on the flux surface. These would govern a gradual increase of $\delta T_{i}$ via parallel transport. When averaged on the flux surface these avalanches would interfere to produce a weak background heat flux. e The temperature variation also provides an estimate of the fluctuation level, Fig. 19. One finds that $\tilde{T}_{i}$ is of the order of $1.510^{-2} T_{0}$ leading to $\tilde{T}_{i} / T_{i}$ of the order of $1 \%$. Since $\tilde{T}_{i}$ is rather homogeneous in the domain of interest, one thus finds that $\tilde{T}_{i} / T_{i}$ increases towards the edge like $1 / T_{i}$. This fluctuation level is estimated for the flux surface averaged temperature, so that local events can have much large magnitude.

When considering the existence of strong peaks of the temperature variation $\delta \tilde{T}_{i}$, there is a possibility of temperature gradient inversion. On Fig. 19 left hand side the linear temperature profile associated to the typical gradient is indicated. The latter is comparable to the largest gradients developed in the peaks of $\delta T_{i}$. One thus finds that in the region where $\delta T_{i}$ is increasing, it can balance the global temperature gradient and lead to a region with a flat temperature profile. In the other region, where $\delta T_{i}$ is decreasing, it can double the temperature gradient.

\subsection{Poloidal distribution of the electric drift velocity patterns}

The available flux averaged quantities such as the ion heat flux or the temperature field do not give precise information on the location of the structures and consequently on the mechanisms that drive the self organisation. Of particular interest is the avalanche transport across the micro-barriers and the occurrence of radially localised corrugation disruptions that also govern the reorganisation of the corrugation pattern. The most detailed information we have is that of the electric potential. However, the correlation between this field and actual avalanches is not straightforward. Indeed, the electric field determines the channel followed by the avalanches but only the correlation between such channels and the temperature field fluctuations determines the actual avalanche is terms of ballistic heat flux transport events.

Given the electric potential field, they are two means to investigate avalanche signatures, one is to track regions with rapid evolution of the structure, as in Section 5 , the other is to track patterns that are correlated to avalanche transport such as large magnitude radial ExB velocity, and in the case of avalanche shearing to large magnitude poloidal ExB velocity as well as large Reynold stress. In particular, one might expect that barrier disruptions depend on the amplitude of the radial velocity of the avalanches. Indeed, the latter could be a criterion determining the probability for an avalanche to cross the micro-barrier, the larger the radial velocity, the less time the avalanche experiences the shearing effect and the more likely it will burn through the barrier. Furthermore one can expect a decrease of the radial convection velocity across the micro-barrier, avalanche slowing down effect. However, this simple mechanism does not appear to match the simulation evidence:

- the maximum radial velocity in the region prior to a micro-barrier is not correlated to the disruptive features of the micro-barrier,

- the radial velocity does not always decrease after a transport barrier,

It thus appears that the magnitude of the radial velocity is not the appropriate criteria to determine the avalanches that will trigger a disruption of the corrugation structure. Conversely, the Reynolds stress is found to be more sensitive in identifying disruptive bursts. However, a correlation appears to be rather complex and difficult to assess.

We investigate here the distribution of the radial and poloidal velocity along $\theta$ averaged on a radial interval $\Delta r_{u}$ upstream (towards the heat source) of the observed barrier. From Fig. 20 we notice that the large values of the poloidal velocity are not homogeneously distributed in the $\theta$-plane. Depending on the time interval, the large magnitude velocity fluctuations are mostly localised either above or below the low-field-side midplane, hence they do not exhibit top-down symmetry. Regarding the radial velocity, one finds that the distribution of the largest values is more symmetric, hence with top-down symmetry, and thus centred towards $\theta \approx 0$.

Tracking the location of the maximum values of the poloidal velocity in the $\theta$-plane we obtain the pattern displayed on Fig. 21. One can identify on this plot structures that are localised radially and that last on mesotime scales. These structures are reminiscent of those of the zonal flow, Fig. 5. Comparing the profile of this structure with the zonal flow shear (averaged on a time window ranging from 3900 to $\left.4000 a / c_{0}\right)$, Fig. 22, one finds that these two fields are modulated with opposite signs. Furthermore, one finds that the poloidal location of the maxima exhibit a rather square shaped modulation. This 

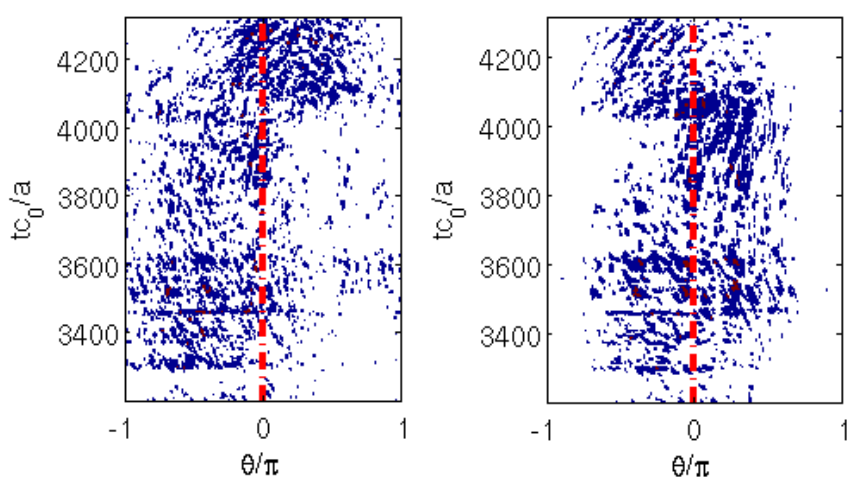

Fig. 20. Left hand side, root mean square of the poloidal velocity at $\Delta r_{u},(2)$. Right hand side, root mean square of the radial velocity at $\Delta r_{u}$

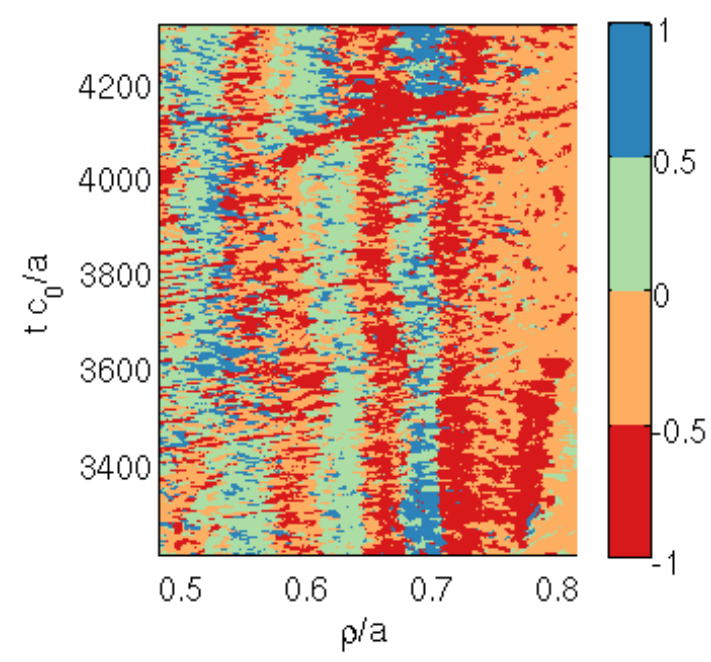

Fig. 21. Time trace of the profile of the localisation of the maxima of the poloidal velocity. The colour scale represents the position $\theta / \pi$ of the maximum of the poloidal velocity at a given time and radial position.

suggest a transition between two patterns with opposite poloidal locations.

\subsection{Up-down asymmetry of the stopping capability of the micro-barriers}

Given the up-down asymmetry of the poloidal electric drift velocity of the fluctuations, we can reconsider the effect of corrugations on avalanches when taking into account the poloidal location. The disruption phenomena would then not only be due to the velocity amplitude of avalanches but would also depend on the localisation of the interaction between the two structures in the poloidal plane.

In Ref.[31], the stopping capability of a barrier is assumed to be governed by the slowing down of the radial motion. When addressing this issue, we find that the be-

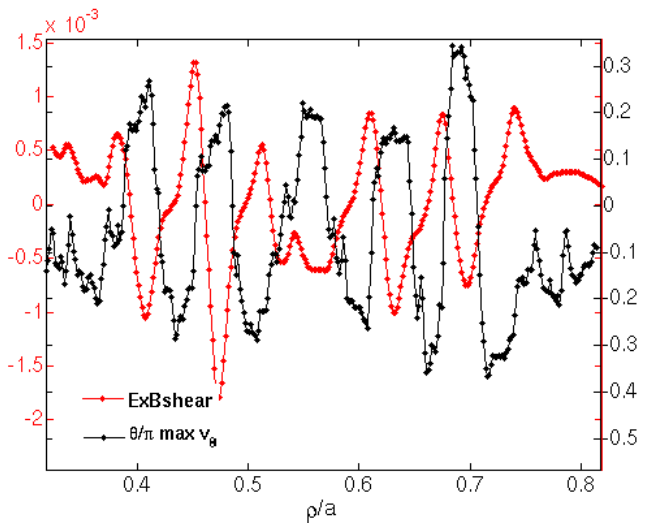

Fig. 22. Profiles of the localisation of the maxima of the poloidal velocity and of the zonal flow shear.

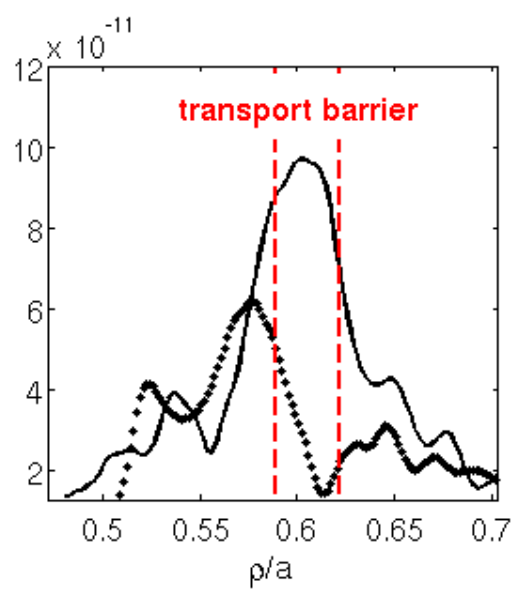

Fig. 23. Variation of the radial velocity along $r$ with up-down asymmetry. Plain line bottom region $p i \leq \theta \leq 2 \pi$, dashed line upper region $0 \leq \theta \leq \pi$.

haviour of the radial drift velocity depends on the location in the poloidal plane, fig.23. More precisely, we observe that the radial velocity decreases at the barrier location when the interaction in poloidally localised in the upper region $0 \leq \theta \leq \pi$. Conversely, there is no noticeable effect when the interaction is localised in the lower region so that $\pi \leq \theta \leq 2 \pi$. Furthermore, when plotting the amplitude of the radial velocity fluctuations in a contour plot of radial profiles versus time, Fig. 24, left hand side panel)), one cannot identify a clear cut effect of the transport barrier (at the dashed vertical lines) unlike the strong effect on the turbulent heat flux Fig. 16 and Fig. 17. However, when splitting the data into the upper and lower regions, we find that the corrugation exhibits a moderate stopping capability whenever $\pi \leq \theta \leq 2 \pi$, hence in the lower region, middle panel of Fig. 24, and a stronger one for $0 \leq \theta \leq \pi$, in the upper region, right hand side panel of Fig. 24.

Provided one considers that the large magnitude of the fluctuations of the radial and poloidal electric drift 

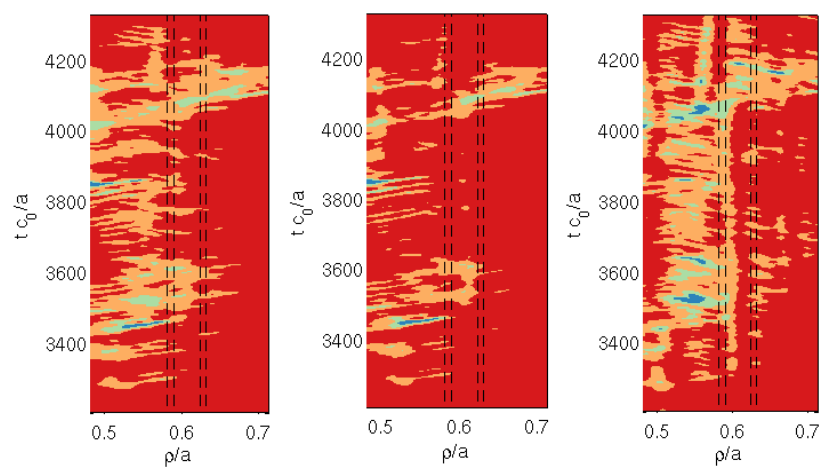

Fig. 24. Contour plot of the amplitude of the radial velocity versus radius and time. Left hand side panel for all values of thet $a$, middle panel for the bottom region $\pi \leq \theta \leq 2 \pi$, and for the top region $0 \leq \theta \leq \pi$ right panel.

velocities occur at the avalanche location, one can build the following picture:

- the maximum poloidal velocity is localised in regions with maximum zonal flow shear, which suggests a maximum stretching of the avalanche structure at the shear layers, in agreement with the standard image of shear layers.

- the region with maximum stretching appears to flip from up above the midplane on the low field side $\theta / \pi \approx$ $1 / 4$, to down, below the miplane on the low field side $\theta / \pi \approx-1 / 4$. This suggests a dependence of the localisation with the sign of the zonal flow and a moderate effect of the magnetic shear.

- the times with efficient stretching correspond to times with effective stopping capability of the micro-barriers for avalanches interacting with the barrier at the poloidal location of maximum avalanche stretching.

Depending on the sign of the zonal flow, the avalanches, which are born on the low field side in the region $[-\pi / 4, \pi / 4]$ drift poloidally. The maximum stopping capability of the micro-barriers occurs where the poloidal stretching is maximum and the outward radial velocity minimum. The clear impact of this process on heat turbulent transport is in agreement with the dominant role of avalanches in the heat transport process. The latter is found to be regulated by the self generated micro-barriers. It is also found to be localised poloidally and toroidally.

\section{Dynamics of marginally trapped \& passing particles and their impact on the distribution function}

The analysis of the GYSELA simulations presented in the previous Sections only use the first moments of the distribution, namely the density used to determine the electric field Eqs. (5a, 5b) and the temperature that also yields the heat flux. We are interested here in completing the description of this physics by considering specific signatures of the kinetic framework. The particle trajectories

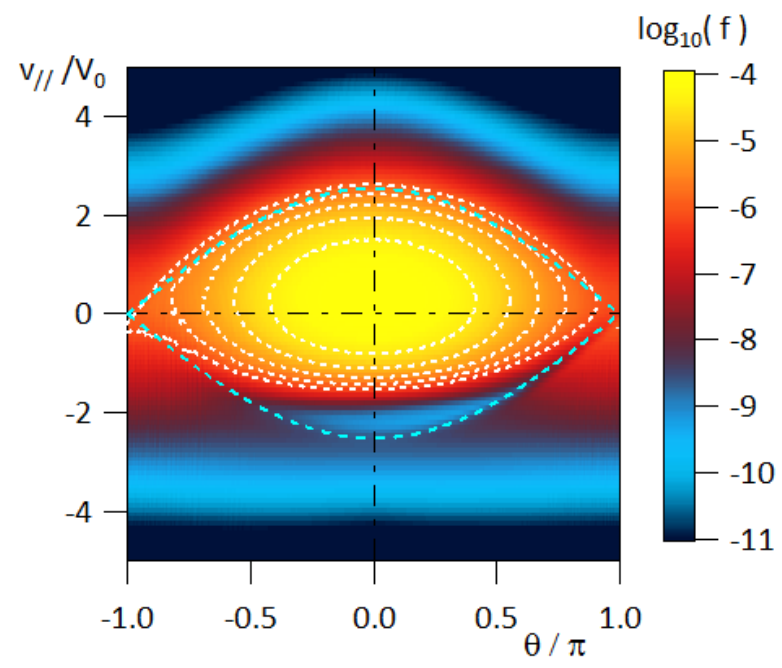

Fig. 25. Distribution function (log scale) in the $\left(\theta, v_{\|}\right)$plane for a large value of $\mu$ and of the inverse aspect ratio. The dashed line is the standard island separatrix. Dotted white contours for trapped particles are also indicated.

(2a, 2b) that are explicitly used in the Vlasov equation (1) can be associated to the Hamiltonian $H_{e q}$ :

$$
\begin{aligned}
H_{e q} & =\frac{1}{2} m v_{\|}^{2}+\mu B \quad ; \quad \mu=\frac{m v_{\perp}}{2 B} \\
B & =\frac{R_{0} B_{0}}{R} \simeq B_{0}\left(1-\frac{r}{R_{0}} \cos (\theta)\right)
\end{aligned}
$$

The variation of the amplitude of the magnetic field in the phase space then governs the separation of particles between trapped and passing depending on the occurrence l of a sign reversal of $v_{\|}$along the particle trajectories: if $v_{\|} \neq 0 \forall \theta \in[0,2 \pi]$, along the trajectory, the particle is "passing", conversely for "trapped particles" $v_{\|}=0$ at two points of the trajectory. With the approximation of the magnitude of the magnetic field in Eq.(15b), the system is equivalent to that of the pendulum. Provided the tokamak axisymmetry is preserved, the toroidal angular momentum is an invariant. Using this constraint one can readily show that any change in $v_{\|}$is associated to a radial displacement. For trapped particles, the reversal of $v_{\|}$ then governs a radial excursion of the trajectory leading to the idea of "banana shape orbits". The typical width of these orbits is $\left.\delta_{b} \approx \rho_{i} q / \sqrt{(} \epsilon\right)$, where $\rho_{i}$ is the ion Larmor radius related to $\mu, q$ is the safety factor Eq.(7) and $\epsilon=a / R_{0}$ the inverse aspect ratio. This feature underlines the departure from the reference pendulum model since $v_{\|}$ is not an invariant of the system when the perturbation $\mu B=0$.

In a first approximation, trapped particles thus exhibit a parallel bounce motion between two turning points together with a $\delta_{b}$ variation of its radial position. To complete this description, it can also be shown that trapped particles exhibit a slow frequency drift of their toroidal location [32]. Furthermore, close to the boundary between trapped and passing, the trapped particles exhibit an in- 


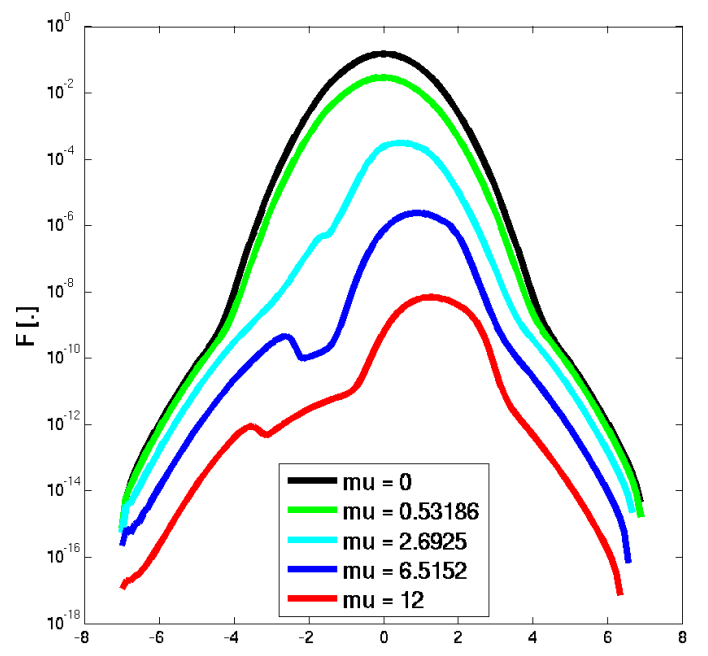

Fig. 26. Cut of the distribution of guiding centers at $r / a \sim$ $0,85, \theta=\pi / 2, \phi=0$. For high values of $\mu$ one can observe a significant departure from a Maxwellian distribution. The transition from trapped to passing particles is reminiscent of neoclassical effects.

version of this drift frequency [32]. Finally, trapped particles have a negligible contribution to the current as well as to the plasma momentum due to the averaging out by the bounce motion. Provided the collision mean free path is large compared to the size of the trapped orbits, the properties of the trapped particles remain unchanged while the passing particle exhibit a poloidal rotation proportional to the radial temperature gradient. A matching layer then builds-up in the distribution function at the trapped-passing boundary [17].

When the axisymmetry of the trajectories is broken by turbulence, the topological separation between passing and trapped particles can be expected to be modified. However the electrostatic energy to be added in the Hamiltonian Eq.(15a) is of order $\rho_{*}$. The turbulence will therefore mostly modify the particle trajectories that remain in phase with the perturbation. The stagnation point of the trapped particle, $\theta=\pi, v_{\|}=0$, that corresponds to the hyperbolic or X-point of the pendulum phase space, will thus be most sensitive to low frequency perturbations. The latter can then modify the distribution function in particular in the vicinity of the trapped-passing matching layer.

Let us first consider the distribution function in $\theta, v_{\|}$ planes where at lowest order approximation one expects to recover the pendulum phase portrait, the island pattern. Since the width of trapped region scales like $\left(\mu B_{0} / T_{0}\right)^{1 / 2}$ we first consider $\mu=6.5 T_{0} / B_{0}$, a rather large value. Similarly, one selects a radial position such that $\epsilon \approx 0.246$ is also large $(\epsilon \leq 0.30)$. As zero order, one recovers the expected island structure, Fig. 25. The changes with respect to the latter seem to be localised at $v_{\|} \leq 0$. In particular, one readily observes a pattern with a dip in the vicinity of the upper separatrix and an enhanced probability density

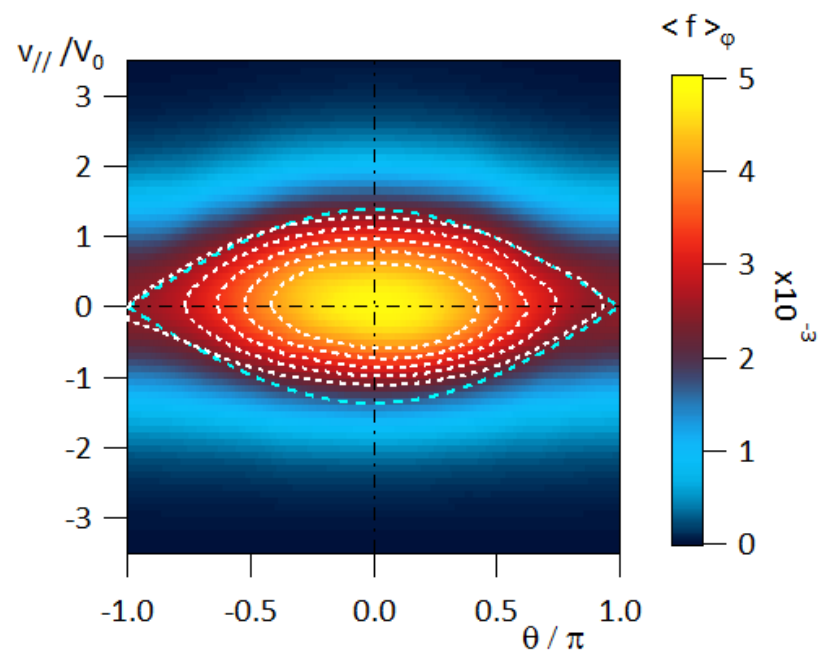

Fig. 27. Toroidally averaged distribution function $<f>\varphi$ (linear scale) in the $\left(\theta, v_{\|}\right)$plane for value $\mu \approx 2$ and at midradius of the simulation region. The dashed line is the standard island separatrix. Dotted white contours for trapped particles are also indicated.

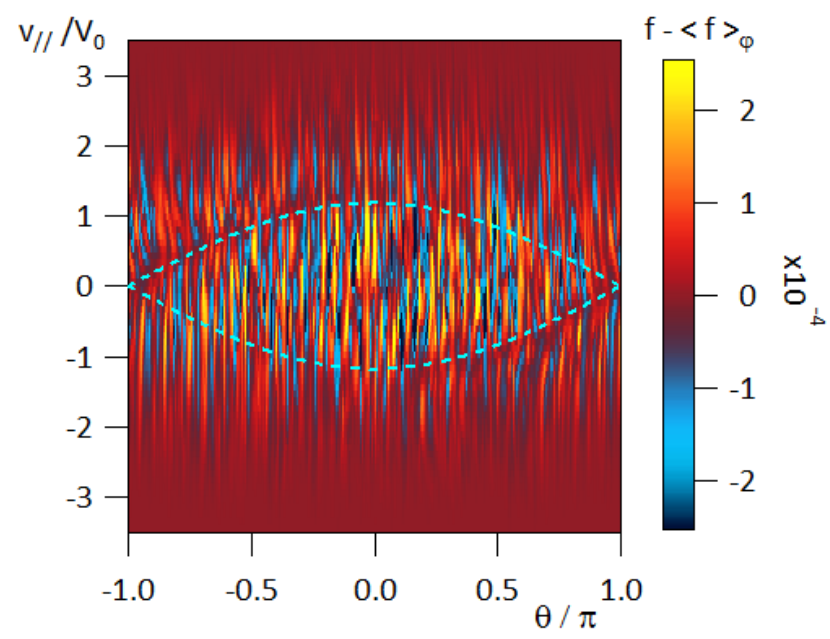

Fig. 28. Fluctuation of the distribution function $f-<f>\varphi$ (linear scale) in the $\left(\theta, v_{\|}\right)$plane for value $\mu \approx 2$ and at midradius of the simulation region. The dashed line is the standard island separatrix.

above the X-point. This pattern exhibits a weak dependence on the toroidal angle $\varphi$ and can be thus considered as axisymmetric. This first order modification of the island pattern appears to decrease with magnetic moment $\mu$, Fig. 26. This distortion of the distribution function in phase-space $\left(v_{\|}, \theta\right)$ is also quite clear when analysing the distribution as a function of $v_{\|}$for different values of $\mu$, Fig. 26. Since the $E \times B$ drift velocity does not depend on the particle energy while the vertical drift does Eq.(3b), one can consider that this structure is governed by neoclassical effects. 
As already stressed, this neoclassical effect is less stringent at lower $\mu$ and at reduced aspect ratio, hence smaller radius, Fig. 27. This allows one to properly locate the separatrix between the passing and trapped particles, Fig. 27. Using this data one can then consider the fluctuations of the distribution function, Fig. 28. These are defined as the difference between the toroidally averaged distribution function $<f>_{\varphi}$ as plotted on Fig. 27 and the actual distribution function at $\varphi=0$. One can notice that the fluctuations are localised in $\theta$ and elongated in $v_{\|}$. There seems to be little difference between the trapped and passing particles, but for two regions where the fluctuations appear to be damped, along the separatrix $v_{\|}>0, \theta<0$ and $v_{\|}<0, \theta>0$, Fig. 28. A closer look also indicates that the fluctuations of the distribution function is these regions are slightly distorted when compared to the two other regions where the fluctuations are well aligned along the $v_{\|}=$constant vertical lines.

\section{Discussion and conclusion}

The simulation effort to recover the flat top conditions of Tore Supra shot TS45511 with the flux driven and global code GYSELA has led us to investigate a parameter space that appears to stand rather close to marginality, leading to a slow transient. The latter is characterised by self organisation and in particular by micro-barriers that tend to slow the evolution. The long simulations times in this regime offer an opportunity to investigate the self-organisation of structures from typically one Larmor radius $\approx \rho_{0}$ to the full radius of the simulation region $\approx 250 \rho_{0}$. With respect to time, the shortest time that is available in the output data, the diagnostic time $t_{\text {diag }}$ appears to be too long to resolve some of the events, while the longest time, that is required to reach the steady state is not yet reached. This leads to a range of characteristic times from $\leq t_{\text {diag }} \approx 0.8 a / c_{0}$ up to $\geq 7200 a / c_{0}$. The corresponding amount of data in $5 \mathrm{D}$ cannot be stored and we have been led to analyse these simulations using existing diagnostics, the most detailed being the electric potential in $2 \mathrm{D}+$ time at given toroidal position. We have also considered flux-averaged fields such as the ion temperature of the heat flux. The full 5D distribution function that we have analysed in Section 7 is only available at a couple of given times (restart conditions). The conclusion of our analysis have to be set in this perspective, which combines very large data handling but incomplete description, as well as long simulation time but still insufficient statistics. These are required to capture significantly the events that prevail in the heavy tails that characterise turbulence self-organisation [33].

With the experience of $2 \mathrm{D}$, slab and fluid simulations assuming invariance along the field line $[8,25]$ a fascinating issue is to determine the 3 -D structure of the avalanches that are reported in gyrokinetic simulations $[5,15,26]$. In Section 5 we analyse the structure of the bursts of the electrostatic potential. We concentrate on the bursts that have a magnitude larger than 2 r.m.s.. We find evidence of parallel expansion and retraction with parallel velocities of the order of $c_{0}$, the ion reference thermal velocity. The maximum toroidal extent is found to be of the order of $0.8 \pi$ and lasts typically $\tau_{\|} \approx 5 a / c_{0}$. These bursts are localised towards the low field side midplane and exhibit an up-down asymmetry that flips from up to down and vice versa in time. The ballooned pattern of such a structure is typically $\pm \pi / 4$ in the poloidal plane, in agreement with available experimental observations [27-29].

Using the electrostatic potential in the $\varphi=0$ poloidal plane, one can investigate the properties of its poloidal modes. The two first modes $m=0$ and $m=1$ exhibit a global structure in the radial direction with respectively zero and one sign change. The magnitude of the $m=0$ mode is 100 times that of the $m=1 \operatorname{mode}\left(1 / \rho_{*}=300\right)$ so that the poloidal shear layers are governed by the $m=0$ mode, hence the zonal flows. These are therefore poloidally and toroidally symmetric. It is noticed, but not understood, that the $m=0$ exhibits spikes that are characterised by fast radial propagation, of the order of the ion thermal velocity $c_{0}$. These look like the so-called "gong"modes [21, 22] although completely different in nature. In contrast to the $m=0$ and $m=1$ the higher mode number amplitudes have a complex pattern but exhibit little sign of radial localisation. Shear layers, and the occurrence of rational surfaces along the profile of the safety factor do not seem to govern strong localisation of these modes. However, one finds that the phase of these modes is correlated to the local (in radius and time) zonal flow velocity. More strikingly, one finds that the pulsation exhibits a linear dependence on the mode number. This dispersion-like property can be attributed to a Doppler effect essentially governed by the zonal flow. This provides an experimental means to measure the zonal flow velocities and their possible reversal. The intrinsic mode pulsation, estimated from the linear analysis and proportional to the density diamagnetic frequency [5] could not be assessed.

The micro-barriers, also called corrugations or staircases [16], have been investigated using the ratio of turbulent over total heat flux [31]. One finds that the corrugation structures are complex and can be considered as a combination the two shear layers on either side of a peak, positive or negative, of the zonal flow velocity. This aspect is supported by the behaviour of the ion temperature fluctuations. One observes two scales for these fluctuations. The small scale fluctuations appear to bounce between the shear layers but cannot penetrate into the corrugations. The large fluctuations, correlated to the heat flux avalanches can disrupt or displace the corrugation and experience therefore longer ballistic transport events. They are also associated to enhanced fluctuation levels and larger heat fluxes. Both scales of fluctuations propagate at the same velocities, of the order of $0.5 \rho_{*} c_{0}$. The typical size of the corrugations, hence of the peak of the zonal flow velocity, is found to be of the order of $\approx 3 \rho_{0}$. As a consequence, the characteristic transverse time to cross a corrugation is $\tau_{\perp} \approx 6 a / c_{s}$. One finds there- 
fore that the expansion time in the parallel direction, $\tau_{\|}$, which bounds the filament structure, is comparable to the transverse time scale to cross a corrugation, $\tau_{\|} \approx \tau_{\perp}$. This might be an important issue in understanding the complex interplay between avalanches, filaments, corrugations and ballooned transport.

The analysis of the stopping capability of the avalanches by the corrugations has indicated an up-down asymmetry. Depending on the sign of the shear of the zonal flow, the maxima of the poloidal velocity is located either above or below the midplane. The stopping capability of the corrugations is also found to exhibit such up-down asymmetry. This evidence is presently investigated to clarify the stopping capability of avalanches by the corrugations as well as the corrugation disruptive features possibly induced by the avalanches (in the spirit of [31]).

Kinetic features complete the analysis of the phase space patterns. The largest impact of kinetics is found to be the island structure that defines trapped and passing particles. The zero order of this structure is recovered towards the core. Further to the edge, the interplay with the drifts, and thus the neoclassical effects, govern a distortion of the island and significant departure from the Maxwellian distribution despite the large edge collisionality [17]. This first order effect can be related to the evaporation of the fastest trapped particles as well as a transfer of momentum from the trapped to the passing particles due to either turbulence or collisions. The third order effect is that associated to turbulence. It plays a role for the same class of particles as that contributing to the neoclassical transport leading to interaction between these two physics. The importance and magnitude of the latter remains to be assessed.

The long simulation of the Tore Supra shot TS45511 in a regime close to marginality have disclosed complex self-organisation with signatures of local events: avalanche transport, corrugations, that we identify as a zonal flow velocity peak together with the shear regions on either side, as well as large scale organisation captured by Fourier modes. The main mode is the zonal flow pattern determined by the 0-mode of the poloidal spectrum. Further investigation with $3 \mathrm{D}$ and $5 \mathrm{D}$ data sets when available is necessary and even longer simulation runs will be required to complete both the analysis and the statistics.

Acknowledgments. The authors acknowledge the fruitful and lively discussions held during the Festival de Théorie in Aix-en-Provence. They are most indebted to Pat Diamond for his strong support and very active scientific participation to this event. They are also grateful to Hyeon Park for bringing to their attention his experimental investigation od dispersion relations. This work is carried out in the framework of the ANR-GYPSI and ANR-ESPOIR projects (ANR-10-BLAN-941 and ANR-09-BLAN-0035$01)$.

\section{References}

1. M. Shimada, D. Campbell, V. Mukhovatov, M. Fujiwara, N. Kirneva, K. Lackner, M. Nagami, V. Pustovitov, N. Uckan, J. Wesley, N. Asakura, A. Costley, A. Donn, E. Doyle, A. Fasoli, C. Gormezano, Y. Gribov, O. Gruber, T. Hender, W. Houlberg, S. Ide, Y. Kamada, A. Leonard, B. Lipschultz, A. Loarte, K. Miyamoto, V. Mukhovatov, T. Osborne, A. Polevoi, and A. Sips, "Chapter 1: Overview and summary," Nuclear Fusion, vol. 47, no. 6, p. S1, 2007.

2. X. Garbet, Y. Idomura, L. Villard, and T. Watanabe, "Gyrokinetic simulations of turbulent transport," $\mathrm{Nu}$ clear Fusion, vol. 50, no. 4, p. 043002, 2010.

3. A. J. Brizard and T. S. Hahm, "Foundations of nonlinear gyrokinetic theory," Rev. Mod. Phys., vol. 79, pp. 421-468, Apr 2007.

4. V. Grandgirard, Y. Sarazin, P. Angelino, A. Bottino, N. Crouseilles, G. Darmet, G. Dif-Pradalier, X. Garbet, P. Ghendrih, S. Jolliet, G. Latu, E. Sonnendrcker, and L. Villard, "Global full- f gyrokinetic simulations of plasma turbulence," Plasma Physics and Controlled Fusion, vol. 49, no. 12B, p. B173, 2007.

5. Y. Sarazin, V. Grandgirard, J. Abiteboul, S. Allfrey, X. Garbet, P. Ghendrih, G. Latu, A. Strugarek, and G. Dif-Pradalier, "Large scale dynamics in flux driven gyrokinetic turbulence," Nuclear Fusion, vol. 50, no. 5, p. 054004, 2010.

6. B. A. Carreras, D. Newman, V. E. Lynch, and P. H. Diamond, "A model realization of self?organized criticality for plasma confinement," Physics of Plasmas (1994-present), vol. 3, no. 8, pp. 2903-2911, 1996.

7. X. Garbet and R. E. Waltz, "Heat flux driven ion turbulence," Physics of Plasmas (1994-present), vol. 5, no. 8, pp. 2836-2845, 1998.

8. Y. Sarazin and P. Ghendrih, "Intermittent particle transport in two-dimensional edge turbulence," Physics of Plasmas (1994-present), vol. 5, no. 12, pp. 4214-4228, 1998.

9. S. Tokunaga, H. Jhang, S. S. Kim, and P. H. Diamond, "A statistical analysis of avalanching heat transport in stationary enhanced core confinement regimes," Physics of Plasmas (1994-present), vol. 19, no. 9, pp., 2012.

10. P. H. Diamond, S.-I. Itoh, K. Itoh, and T. S. Hahm, "Zonal flows in plasmaa review," Plasma Physics and Controlled Fusion, vol. 47, no. 5, p. R35, 2005.

11. P. Bak, C. Tang, and K. Wiesenfeld, "Self-organized criticality: An explanation of the $1 / \mathrm{i}_{\mathrm{i}} \mathrm{fi}_{\mathrm{i}} / \mathrm{i}_{\mathrm{i}}$, noise," Phys. Rev. Lett., vol. 59, pp. 381-384, Jul 1987.

12. Y. Sarazin, X. Garbet, P. Ghendrih, and S. Benkadda, "Transport due to front propagation in tokamaks," Physics of Plasmas (1994-present), vol. 7, no. 4, pp. 1085-1088, 2000.

13. J. Candy and R. E. Waltz, "Anomalous transport scaling in the diii-d tokamak matched by supercom- 
puter simulation," Phys. Rev. Lett., vol. 91, p. 045001, Jul 2003.

14. B. F. McMillan, S. Jolliet, T. M. Tran, L. Villard, A. Bottino, and P. Angelino, "Avalanchelike bursts in global gyrokinetic simulations," Physics of Plasmas (1994-present), vol. 16, no. 2, pp. -, 2009.

15. Y. Idomura, H. Urano, N. Aiba, and S. Tokuda, "Study of ion turbulent transport and profile formations using global gyrokinetic full- $\mathrm{f}$ vlasov simulation," Nuclear Fusion, vol. 49, no. 6, p. 065029, 2009.

16. G. Dif-Pradalier, P. H. Diamond, V. Grandgirard, Y. Sarazin, J. Abiteboul, X. Garbet, P. Ghendrih, A. Strugarek, S. Ku, and C. S. Chang, "On the validity of the local diffusive paradigm in turbulent plasma transport," Phys. Rev. E, vol. 82, p. 025401, Aug 2010.

17. G. Dif-Pradalier, P. H. Diamond, V. Grandgirard, Y. Sarazin, J. Abiteboul, X. Garbet, P. Ghendrih, G. Latu, A. Strugarek, S. Ku, and C. S. Chang, "Neoclassical physics in full distribution function gyrokinetics," Physics of Plasmas (1994-present), vol. 18, no. 6, pp. -, 2011.

18. J. Abiteboul, X. Garbet, V. Grandgirard, S. J. Allfrey, P. Ghendrih, G. Latu, Y. Sarazin, and A. Strugarek, "Conservation equations and calculation of mean flows in gyrokinetics," Physics of Plasmas (1994present), vol. 18, no. 8, pp. -, 2011.

19. G. Darmet, P. Ghendrih, Y. Sarazin, X. Garbet, and V. Grandgirard, "Intermittency in flux driven kinetic simulations of trapped ion turbulence," Communications in Nonlinear Science and Numerical Simulation, vol. 13 , no. 1 , pp. $53-58,2008$. Vlasovia 2006: The Second International Workshop on the Theory and Applications of the Vlasov Equation.

20. Y. Sarazin, V. Grandgirard, J. Abiteboul, S. Allfrey, X. Garbet, P. Ghendrih, G. Latu, A. Strugarek, G. Dif-Pradalier, P. Diamond, S. Ku, C. Chang, B. McMillan, T. Tran, L. Villard, S. Jolliet, A. Bottino, and P. Angelino, "Predictions on heat transport and plasma rotation from global gyrokinetic simulations," Nuclear Fusion, vol. 51, no. 10, p. 103023, 2011.

21. P. Duperrex, A. Pochelon, A. Edwards, and J. Snipes, "Global sawtooth instability measured by magnetic coils in the jet tokamak," Nuclear Fusion, vol. 32, no. 7, p. 1161, 1992.

22. T. Dudok de Wit, A. Pecquet, J. Vallet, and R. Lima, "The biorthogonal decomposition as a tool for investigating fluctuations in plasmas," Physics of Plasmas (1994-present), vol. 1, no. 10, pp. 3288-3300, 1994.

23. K. R. Sreenivasan, A. Bershadskii, and J. J. Niemela, "Mean wind and its reversal in thermal convection," Phys. Rev. E, vol. 65, p. 056306, May 2002.

24. W. Lee, J. Leem, G. S. Yun, H. K. Park, J. A. Lee, Y. B. Nam, Y. U. Nam, W. H. Ko, J. H. Jeong, Y. S. Bae, H. Park, K. W. Kim, C. W. Domier, and N. C. L. $\mathrm{Jr}$, "Poloidal rotation velocity measurement with an mir system on kstar," Journal of Instrumentation, vol. 8, no. 10, p. C10018, 2013.
25. P. Ghendrih, Y. Sarazin, G. Attuel, S. Benkadda, P. Beyer, G. Falchetto, C. Figarella, X. Garbet, V. Grandgirard, and M. Ottaviani, "Theoretical analysis of the influence of external biasing on long range turbulent transport in the scrape-off layer," Nuclear Fusion, vol. 43, no. 10, p. 1013, 2003.

26. J. Abiteboul, P. Ghendrih, V. Grandgirard, T. Cartier-Michaud, G. Dif-Pradalier, X. Garbet, G. Latu, C. Passeron, Y. Sarazin, A. Strugarek, O. Thomine, and D. Zarzoso, "Turbulent momentum transport in core tokamak plasmas and penetration of scrape-off layer flows," Plasma Physics and Controlled Fusion, vol. 55, no. 7, p. 074001, 2013.

27. N. Fedorczak, J. Gunn, P. Ghendrih, P. MonierGarbet, and A. Pocheau, "Flow generation and intermittent transport in the scrape-off-layer of the tore supra tokamak," Journal of Nuclear Materials, vol. 390391, no. 0, pp. 368 - 371, 2009. Proceedings of the 18th International Conference on Plasma-Surface Interactions in Controlled Fusion Device Proceedings of the 18th International Conference on PlasmaSurface Interactions in Controlled Fusion Device.

28. N. Fedorczak, J. Gunn, P. Ghendrih, G. Ciraolo, H. Bufferand, L. Isoardi, P. Tamain, and P. MonierGarbet, "Experimental investigation on the poloidal extent of the turbulent radial flux in tokamak scrapeoff layer," Journal of Nuclear Materials, vol. 415, no. 1, Supplement, pp. S467 - S470, 2011. Proceedings of the 19th International Conference on PlasmaSurface Interactions in Controlled Fusion.

29. N. Fedorczak, J. P. Gunn, J.-Y. Pascal, P. Ghendrih, G. van Oost, P. Monier-Garbet, and G. R. Tynan, "Electrostatic transport in l-mode scrape-off layer plasmas of tore supra tokamak. ii. transport by fluctuations," Physics of Plasmas (1994-present), vol. 19, no. 7, pp. -, 2012.

30. L. Villard, P. Angelino, A. Bottino, S. J. Allfrey, R. Hatzky, Y. Idomura, O. Sauter, and T. M. Tran, "First principles based simulations of instabilities and turbulence," Plasma Physics and Controlled Fusion, vol. 46, no. 12B, p. B51, 2004.

31. E. Floriani, G. Ciraolo, P. Ghendrih, R. Lima, and Y. Sarazin, "Self-regulation of turbulence bursts and transport barriers," Plasma Physics and Controlled Fusion, vol. 55, no. 9, p. 095012, 2013.

32. B. B. Kadomtsev and O. P. Porgutse, "Turbulence in toroidal systems," Reviews of Plasma Physics, vol. 5, p. 249, 1970.

33. P. Ghendrih, C. Norscini, F. Hasenbeck, G. DifPradalier, J. Abiteboul, T. Cartier-Michaud, X. Garbet, V. Grandgirard, Y. Marandet, Y. Sarazin, P. Tamain, and D. Zarzoso, "Thermodynamical and microscopic properties of turbulent transport in the edge plasma," Journal of Physics: Conference Series, vol. 401, no. 1, p. 012007, 2012. 